\title{
Integrated Analysis of tRNA-Derived Small RNAs in Proliferative Human Aortic Smooth Muscle Cells
}

\author{
Jian-Zhi Zhao \\ Hebei Medical University \\ Jia-Jie Lin \\ Hebei Medical University \\ Miao Gong \\ Hebei Medical University \\ Yu Wang \\ Hebei Medical University \\ Ke-Xin Liu \\ Hebei Medical University \\ Mei-Yang Du \\ Hebei Medical University \\ Huan-Huan Li \\ Hebei Medical University \\ Si-Fan Wang \\ Hebei Medical University \\ Li-Yun Yang \\ Hebei Medical University
}

Bo Sun

Hebei Medical University

Peng Kong

Hebei Medical University

Kun Liu

Hebei Medical University

Bin Li

Hebei Medical University

Shao-Guang Sun ( $\nabla$ sunshaoguang00@163.com )

Hebei Medical University https://orcid.org/0000-0002-2827-0948

Research

Keywords: tsRNA, VSMC, Proliferation, circRNA, p53, MFN2 
Posted Date: May 11th, 2021

DOl: https://doi.org/10.21203/rs.3.rs-482321/v1

License: (c) (i) This work is licensed under a Creative Commons Attribution 4.0 International License. Read Full License 


\section{Abstract \\ Background}

Abnormal proliferation of vascular smooth muscle cells (VSMCs) contributes to vascular remodeling diseases. Recently, it has been discovered that tRNA-derived small RNAs (tsRNAs), a new type of noncoding RNAs, are related to the proliferation and migration of VSMCs. tsRNAs regulate target gene expression through miRNA-like function. This study aims to explore the potential of tsRNAs in human aortic smooth muscle cell (HASMC) proliferation.

\section{Methods}

High-throughput sequencing was performed to analyze the tsRNA expression profile of proliferative and quiescent HASMCs. Quantitative real-time PCR (qRT-PCR) was performed to validate sequence results and subcellular distribution of AS-tDR-001370, AS-tDR-000067, AS-tDR-009512, and AS-tDR-000076. Based on microRNA-like functions of tsRNAs, we predicted target promoters, mRNAs, and circular RNAs (circRNAs), constructed tsRNA-promoter, tsRNA-mRNA, and circRNA-tsRNA interaction networks. Gene Ontology (GO) and Kyoto Encyclopedia of Genes and Genomes (KEGG) pathway analyses were performed to reveal the function of target genes. Western blot and EdU incorporation assays were utilized to detect the effect of tsRNAs on HASMC proliferation.

\section{Results}

Compared with quiescent HASMCs, 887 up-regulated and 951 down-regulated tsRNAs in proliferative HASMCs were identified (fold change $>2$ or $<-2, p$-value $<0.05$ ). AS-tDR-001370, AS-tDR-000067, AS-tDR009512, and AS-tDR-000076 were up-regulated in proliferative HASMCs and were mainly located in the nucleus. Bioinformatics analysis suggested that they involve a variety of terms and pathways related to VSMC proliferation. Knockdown of AS-tDR-000067 promoted the expression of target gene p53 and inhibited HASMC proliferation. Knockdown of AS-tDR-000076 promoted the expression of target gene mitofusin 2 (MFN2) and inhibited HASMC proliferation.

\section{Conclusion}

During HASMC proliferation, the expression levels of many tsRNAs are altered. AS-tDR-000067 and AStDR-000076 may become a new therapeutic target for vascular remodeling diseases.

\section{Introduction}

The death toll of cardiovascular diseases increases year by year, among which ischemic heart disease and cerebrovascular disease account for more than $80 \%$ of the deaths [1]. Vascular smooth muscle cells 
(VSMCs), one of the cell types in blood vessels, mainly contract and relax to maintain blood pressure and flow [2]. Abnormal proliferation of VSMCs is a vital process in the pathogenesis of numerous vascular remodeling diseases, such as atherosclerosis [3], hypertension [4], vascular stenosis [5], and diabetic vascular complications [6]. Therefore, exploration of VSMC proliferation will contribute to the treatment of vascular proliferative diseases.

With the development of deep sequencing technology, tRNA-derived small RNAs (tsRNAs), a new class of small non-coding RNAs derived from tRNAs, have been discovered in various organisms [7]. tsRNAs function on cell phenotype, especially cell proliferation [8-13]. tRF ${ }^{\text {GnCTG }}$, which is highly expressed in the rat common carotid artery (CCA) intimal hyperplasia model, targets FAS cell surface death receptor (FAS) to promote the proliferation and migration of rat VSMCs [14]. In the mouse model of ischemia, tsRNAs derived from tRNA ${ }^{\mathrm{Val}}$ and tRNA ${ }^{\text {Gly }}$ are significantly up-regulated in endothelial cells, thereby inhibiting their proliferation, migration, and tube formation [8]. CU1276, a microRNA (miRNA)-like tsRNA in human mature $B$ cells, is down-regulated in lymphoma cells and attenuates its inhibition of target gene replication protein A1 (RPA1), thereby promoting cell proliferation [9]. In breast cancer cells under hypoxia, a class of tsRNAs derived from tRNA ${ }^{\text {Glu }}$, tRNA ${ }^{A s p}$, tRNA $^{\text {Gly }}$, and tRNA ${ }^{T y r}$ destabilize multiple oncogenic transcripts through competitive RNA-binding protein Y-box binding protein 1 (YBX1) displacement, thereby inhibiting cell proliferation, invasion, and migration [10]. Three 5'-sex hormone-dependent tRNA-

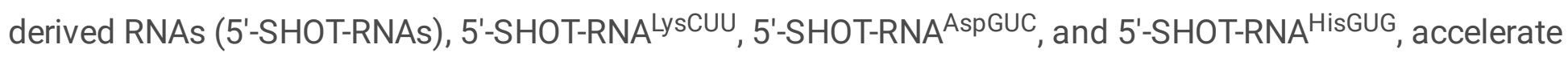
prostate cancer cell proliferation [11]. In addition, cell proliferation-related tsRNAs have also been revealed in other studies $[12,13]$.

Previous studies have shown that tsRNAs regulate various diseases, including cancer [15], alcoholic fatty liver disease [16], viral infection [17], and neurodegenerative diseases [18], mainly by interacting with RNAs or proteins. So far, most studies on tsRNAs have focused on cancer; however, regulation of tsRNAs on VSMC proliferation has not been clearly elucidated yet.

This study focuses on screening tsRNAs related to human aortic smooth muscle cell (HASMC) proliferation. Four differentially expressed tsRNAs (DEtsRNAs) (AS-tDR-001370, AS-tDR-000067, AS-tDR009512, and AS-tDR-000076) were selected for qRT-PCR validation. tsRNA-promoter, tsRNA-mRNA, and circular RNA (circRNA)-tsRNA interaction networks were constructed to facilitate the analysis of four DEtsRNAs. Our data showed that the target genes of four DEtsRNAs were enriched in Gene Ontology (GO) terms and Kyoto Encyclopedia of Genes and Genomes (KEGG) pathways related to cell proliferation. Knockdown of AS-tDR-000067 and AS-tDR-000076 inhibited HASMC proliferation. Our study innovatively elucidates tsRNAs related to HASMC proliferation, which provides novel clues to explore vascular remodeling diseases.

\section{Methods}

\section{Cell culture}


Proliferative HASMCs (ScienCell, California, USA) were induced by smooth muscle cell medium (SMCM, ScienCell) containing $2 \%$ fetal bovine serum (FBS, ScienCell), $1 \% 100 \times$ smooth muscle cell growth supplement (SMCGS, ScienCell), and 1\% $100 \times$ penicillin/streptomycin solution (ScienCell). Quiescent HASMCs were induced by FBS and SMCGS starvation for $24 \mathrm{~h}$. Cells were cultured at $37^{\circ} \mathrm{C}$ in an incubator containing $5 \% \mathrm{CO}_{2}$.

\section{tsRNA-seq libraries preparation and sequencing}

To screen tsRNAs related to HASMC proliferation, we performed high-throughput RNA sequencing of three sets of proliferative and quiescent HASMCs. Total RNA of proliferative and quiescent HASMCs was extracted by RNA Simple Total RNA Kit (TIANGEN, Beijing, China). Total RNA samples were pretreated to eliminate the interference of RNA modification in the construction of small RNA-seq libraries: 1) 3aminoacyl (charged) was deacylated to 3'-OH for 3' adaptor ligation; 2) 3'-cP (2', 3'-cyclic phosphate) was removed to 3'-OH for 3' adaptor ligation; 3) 5'-OH (hydroxyl group) was phosphorylated to 5'-P for 5'adaptor ligation; 4) $\mathrm{m} 1 \mathrm{~A}$ and $\mathrm{m} 3 \mathrm{C}$ were demethylated for effective reverse transcription. Subsequently, tsRNA-seq libraries were constructed using the commercial kit for tsRNA sequencing library preparation (IIlumina, California, USA). The kit includes 3'-adapter and 5'-adapter ligation adaptor ligation, cDNA synthesis, and library PCR amplification. PCR amplified fragments with a size of $135 \sim 160 \mathrm{bp}$ (corresponding to the size range of $15 \sim 40$ nt small RNA) were selected as tsRNA-seq libraries. Finally, the prepared tsRNA-seq libraries were quantified using Agilent 2100 Bioanalyzer (Agilent Technologies, California, USA) and then sequenced using Illumina NextSeq 500 (Illumina). Sequencing was performed by Kangchen Bio-tech (Shanghai, China).

\section{Sequencing data analysis}

Image analysis and base calling were performed by Solexa pipeline v1.8 (Off-Line Base Caller software, v1.8). Valid sequences were preserved by alignment statistical analysis for subsequent tsRNA expression profile analysis and differential expression analysis. Sequencing quality was tested by FastQC software, and NovoAlign software (v2.07.11) was applied to align the trimmed reads (with 5', 3'-adaptor bases removed) with the mature tRNA and pre-tRNA sequences of GtRNAdbb: Genomic tRNA Database (http://gtrnadb.ucsc.edu/). Remaining reads were aligned to the transcriptome including mRNA/rRNA/snRNA/piRNA/snoRNA/miRNA biotypes. Expression profile and differential expression of tsRNAs were calculated based on standardized TPM (Transcripts Per Million).

\section{Quantitative real-time PCR (qRT-PCR) validation}

Total small RNAs (smRNAs) of proliferative and quiescent HASMCs were isolated by MiRcute miRNA Isolation Kit (TIANGEN). Quantity and integrity of total smRNAs were measured by NanoDrop ND-1000 (Thermo Fisher Science, Massachusetts, USA) and 1.2\% agarose gel electrophoresis. Total smRNAs were reverse transcribed by miRcute Plus miRNA First-Strand cDNA Kit (TIANGEN). qRT-PCR was performed using SYBR Green analysis of miRcute Plus miRNA qPCR Kit (TIANGEN). Reaction conditions of all samples were: Initial denaturation at $95^{\circ} \mathrm{C}$ for $10 \mathrm{~min}$, heat denaturation at $95^{\circ} \mathrm{C}$ for $10 \mathrm{~s}$, annealing at $60^{\circ} \mathrm{C}$ for $20 \mathrm{~s}$, and extension at $72^{\circ} \mathrm{C}$ for $10 \mathrm{~s}$, with 40 cycles. All samples were normalized using $\mathrm{U} 6$ as an 
internal control. $2^{-\Delta \Delta C t}$ method was applied to calculate the fold change of expression of tsRNAs; Student's $t$-test was performed for statistical significance. Primers of AS-tDR-001370, AS-tDR-000067, AStDR-009512, AS-tDR-000076, and U6 were shown in Supplementary Table 1.

\section{Nuclear and cytoplasmic RNA extraction}

Nuclear and cytoplasmic components of proliferative HASMCs were isolated using the Nuclear/Cytosol Fractionation Kit (BioVision, California, USA) following the manufacturer's instructions. Extraction, quantification, and integrity detection of smRNAs were consistent with the above. Reaction condition of qRT-PCR was consistent with the above. GAPDH and U6 were applied as positive controls for the cytoplasm and nucleus, respectively. Primers were listed in Supplementary Table 1.

\section{Construction of tsRNA-promoter interaction networks}

Based on seed region (tsRNA nucleotides 2-8), downstream target promoters of four DEtsRNAs (AS-tDR001370, AS-tDR-000067, AS-tDR-009512, and AS-tDR-000076) were predicted by RNAhybrid [19] and MiRanda [20]. To further screen proliferation-related genes containing target promoters, Venn analysis was performed on predicted genes containing target promoters by means of differentially expressed mRNAs (DEmRNAs) in proliferative HASMCs (GSE77279) (fold change $>2$ or $<-2, p$-value $<0.05$ ). Finally, tsRNA-promoter interaction networks were constructed using Cytoscape_v3.7.1.

\section{Construction of tsRNA-mRNA interaction networks}

Downstream target genes of four DEtsRNAs (AS-tDR-001370, AS-tDR-000067, AS-tDR-009512, and AStDR-000076) were predicted by TargetScan [21] and MiRanda [20]. Venn analysis between predicted target genes and DEmRNAs in the proliferative HASMCs (GSE77279) (fold change $<-1.5, p$-value $<0.05$ ) was performed to further obtain target DEmRNAs. Finally, tsRNA-mRNA interaction networks were constructed using Cytoscape_v3.7.1.

\section{Construction of circRNA-tsRNA interaction networks}

Based on the miRNA-like function of tsRNAs, upstream target circRNAs of AS-tDR-001370, AS-tDR000067, AS-tDR-009512, and AS-tDR-000076 were predicted using RNAhybrid [19]. Venn analysis between predicted target circRNAs and differentially expressed circRNAs (DEcircRNAs) in the proliferative HASMCs (GSE77278) (fold change $>2$ or $<-2, p$-value $<0.05$ ) was utilized to further enrich target DEcircRNAs. Finally, circRNA-tsRNA interaction networks were constructed using Cytoscape_v3.7.1.

\section{GO and pathway analyses}

GO and KEGG pathway analyses of the proliferation-related gene sets containing target promoters and proliferation-related target DEmRNA sets were performed to explore the potential of AS-tDR-001370, AStDR-000067, AS-tDR-009512, and AS-tDR-000076 in HASMC proliferation using the DAVID database [22].

\section{Antisense oligonucleotide transfection}


Antisense oligonucleotide (ASO) of AS-tDR-000076 and negative control (NC) were designed and synthesized by GENEWIZ (Beijing, China). According to the procedures of Lipofectamine $3000 \AA$ Transfection Reagent (Invitrogen, California, USA), Lipofectamine 3000 was used to transfect 5,000 ng ASO into HASMCs cultured in a $25 \mathrm{~cm}^{2}$ cell culture flask. After $7 \mathrm{~h}$ of incubation in a $37^{\circ} \mathrm{C}$ and $5 \% \mathrm{CO}_{2}$ humidified incubator, the transfection medium was replaced with fresh medium and cultured to the appropriate time point. Sequences of NC and ASO were listed in Supplementary Table 2.

\section{Western blot analysis}

Total protein in HASMCs transfected with ASO or NC for $48 \mathrm{~h}$ was extracted using RIPA buffer (Solarbio, Beijing, China) and $1 \mathrm{mM}$ PMSF (Solarbio). Protein concentration was detected by the Bradford method (Solarbio). Equal amounts of protein were separated by SDS-PAGE and transferred onto polyvinylidene fluoride (PVDF) membranes (Merck, Darmstadt, Germany). At $37^{\circ} \mathrm{C}, 5 \%$ skimmed milk was used to block the membrane for $2 \mathrm{~h}$, followed by washing with Tris-buffered saline (TBS), and then incubated overnight at $4^{\circ} \mathrm{C}$ with the following specific primary antibodies: MFN2 (dilution at a 1: 500, Abcam), GAPDH (dilution at a 1: 1000, Wanleibio). After washing three times in Tris-buffered saline within Tween 20 (TBST) for $5 \mathrm{~min}$, the membrane was incubated in goat anti-rabbit secondary antibody (dilution at a 1: 20,000, Sino Biological) for $1 \mathrm{~h}$ at room temperature. Subsequently, ChemiDoc ${ }^{\mathrm{TM}}$ MP Imaging System (BIO-RAD) visualized the protein signals and quantified band strength.

\section{Cell proliferation analysis}

BeyoClick ${ }^{\mathrm{TM}}$ EdU Cell Proliferation Kit with Alexa Fluor 594 (Beyotime, Shanghai, China) was used for the 5-ethynyl-2'-deoxyuridine (EdU) incorporation assay. Proliferative HASMCs were seeded into 24-well plates, $420 \mathrm{ng}$ ASO or NC were transfected into each well. Proliferative HASMCs were incubated with SMCM containing $2 \% \mathrm{FBS}, 1 \% 100 \times$ SMCGS, and $1 \% 100 \times$ penicillin/streptomycin solution for $48 \mathrm{~h}$ in a humidified chamber containing $5 \% \mathrm{CO}_{2}$ and $95 \% \mathrm{O}_{2}$ at $37^{\circ} \mathrm{C}$. Representative images were obtained through OLYMPUS IX71 (OLYMPUS, Tokyo, Japan).

\section{Statistical analysis}

All data are from at least three independent experiments, expressed in the form of mean \pm standard deviation (SD). Student's $t$-test was performed to compare the differences between the two groups. When $p$-value $<0.05$, the difference was statistically significant.

\section{Results}

\section{Analysis of tsRNA expression profiling}

To screen for tsRNAs related to HASMC proliferation, we performed high-throughput RNA sequencing of three sets of proliferative and quiescent HASMCs. A total of 3,891 tsRNAs were identified by sequencing, of which 887 and 951 tsRNAs were up-regulated and down-regulated in proliferative HASMCs compared to quiescent HASMCs, respectively (fold change $>2$ or $<-2$, $p$-value $<0.05$ ). Scatter plots (Fig. 1a), volcano 
plots (Fig. 1b), and hierarchical clustering (Fig. 1c) were performed to describe the tsRNA expression profiling. According to the fold change and expression abundance, four DEtsRNAs (AS-tDR-001370, AStDR-000067, AS-tDR-009512, and AS-tDR-000076) were selected for qRT-PCR. Consistent with the sequencing, AS-tDR-001370, AS-tDR-000067, AS-tDR-009512, and AS-tDR-000076 were up-regulated in proliferative HASMCs (Fig. 1d). To evaluate the subcellular localization of tsRNA, the nuclear and cytoplasmic components of proliferative HASMCs were separated. Four DEtsRNAs (AS-tDR-001370, AStDR-000067, AS-tDR-009512, and AS-tDR-000076) were mainly located in the nucleus, as confirmed by qRT-PCR (Fig. 1e).

\section{Construction of tsRNA-promoter interaction networks}

Function of tsRNAs binding to Argonaute (AGO) protein is similar to miRNAs [23]. Binding of nuclear miRNAs to promoters leads to activate [24-26] or silence [27-29] the transcription of target genes. Therefore, we speculate that tsRNAs are similar to miRNAs and play a regulatory role through targeting promoters. Four DEtsRNAs (AS-tDR-001370, AS-tDR-000067, AS-tDR-009512, and AS-tDR-000076) were selected to construct tsRNA-promoter interaction networks (Fig. 2). From the networks, AS-TDR-001370, AS-TDR-000067, AS-TDR-009512, and AS-TDR-000076 might target 243, 855, 202, and 120 proliferationrelated genes in a promoter targeted manner, respectively (Fig. 2).

\section{Functional annotation of genes containing target promoters of tsRNAs}

GO and KEGG pathway analyses of target promoters were performed to explore the potential of AS-tDR001370, AS-tDR-000067, AS-tDR-009512, and AS-tDR-000076 in HASMC proliferation using DAVID database [22]. GO analysis revealed that four molecules were enriched in the $\mathrm{GO}$ terms related to cell proliferation. Cell proliferation-related genes containing target promoters of AS-tDR-001370, such as cyclin D1 (CCND1) [30], sprouty RTK signaling antagonist 2 (SPRY2) [31], and bone morphogenetic protein receptor type 2 (BMPR2) [32] were enriched in histone deacetylase binding (ontology: molecular function, GO: 0042826), peptidyl-threonine phosphorylation (ontology: biological process, G0:0018107), and regulation of developmental growth (ontology: biological process, GO: 0048638), respectively (Fig. 3a). Cell proliferation-related genes containing target promoters of AS-tDR-000067, such as Yes1 associated transcriptional regulator (YAP1) [33], high mobility group AT-hook 2 (HMGA2) [34], cyclin dependent kinase 6 (CDK6) [35], and autophagy related 4B cysteine peptidase (ATG4B) [36] were enriched in hippo signaling (ontology: biological process, GO: 0035329), regulation of stem cell population maintenance (ontology: biological process, GO: 2000036), cyclin-dependent protein serine/threonine kinase activity (ontology: molecular function, GO: 0004693), and cysteine-type endopeptidase activity (ontology: molecular function, GO: 0004197), respectively (Fig. 3c). Cell proliferation-related genes containing target promoters of AS-tDR-009512, such as nuclear receptor subfamily 2 group F member 1 (NR2F1) [37], angiogenic factor with G-patch and FHA domains 1 (AGGF1) [38], caldesmon 1 (CALD1) [39], and reticulon 4 (RTN4) [40] were enriched in negative regulation of kinase activity (ontology: biological process, GO: 0033673), regulation of angiogenesis (ontology: biological process, GO: 0045765), actin cytoskeleton (ontology: cellular component, GO: 0015629), and regulation of cell death 
(ontology: biological process, G0: 0010941), respectively (Fig. 3e). Cell proliferation-related genes containing target promoters of AS-tDR-000076, such as CCND1 [41], secreted frizzled related protein 1 (SFRP1) [42], and transforming growth factor beta 1 (TGFB1) [43] were enriched in regulation of cell cycle arrest (ontology: biological process, GO: 0071156), negative regulation of cell growth (ontology: biological process, GO: 0030308), and Notch signaling pathway (ontology: biological process, GO: 0007219), respectively (Fig. 3g).

KEGG analysis revealed that four molecules were enriched in cell proliferation-related pathways. Cell proliferation-related genes containing target promoters of AS-tDR-001370, such as RELA proto-oncogene (RELA) [44], fibroblast growth factor 9 (FGF9) [45], and calcium voltage-gated channel subunit alpha1 G (CACNA1G) [46] were enriched in MAPK signaling pathway (hsa04010) (Fig. 3b). Cell proliferation-related genes containing target promoters of AS-tDR-000067, such as cyclin D3 (CCND3) [47], p53 [48], CDK6 [35], and cyclin dependent kinase 2 (CDK2) ${ }^{[49]}$ were enriched in p53 signaling pathway (hsa04115) (Fig. 3d). Cell proliferation-related genes containing target promoters of AS-tDR-009512, such as CALD1 [39] and inositol 1,4,5-trisphosphate receptor type 1 (ITPR1) [50] were enriched in vascular smooth muscle contraction (hsa04270) (Fig. 3f). Cell proliferation-related genes containing target promoters of AS-tDR000076, such as calcium/calmodulin dependent protein kinase II delta (CAMK2D) [51], cyclin D2 (CCND2) [52], and mitogen-activated protein kinase 9 (MAPK9) [53] were enriched in Wnt signaling pathway (hsa04310) (Fig. 3h).

\section{Construction of tsRNA-mRNA interaction networks}

Many studies have shown that tsRNAs possess miRNA-like functions (that is, down-regulate the expression of target genes in a sequence-dependent manner by binding to AGO) [9, 54, 55]. To explore whether tsRNAs also play a biological role in HASMCs through this mechanism, four DEtsRNAs (AS-tDR001370, AS-tDR-000067, AS-tDR-009512, and AS-tDR-000076) were selected to construct tsRNA-mRNA interaction networks (Fig. 4). From the interaction networks, AS-tDR-001370, AS-tDR-000067, AS-tDR009512, and AS-tDR-000076 might target 46, 69, 184, and 306 DEmRNAs, respectively (Fig. 4).

\section{Functional annotation of target DEmRNAs of tsRNAs}

GO and KEGG pathway analyses of four target DEmRNAs sets were performed to explore the potential of AS-tDR-001370, AS-tDR-000067, AS-tDR-009512, and AS-tDR-000076 in HASMC proliferation [22]. G0 analysis revealed that four molecules were involved in cell proliferation-related $\mathrm{GO}$ terms. Cell proliferation-related target DEmRNA prohibitin 2 (PHB2) [56] of AS-tDR-001370 was enriched in regulation of complement activation (ontology: biological process, GO: 0030449) (Fig. 5a). Cell proliferation-related target DEmRNAs ataxin 3 (ATXN3)[57] and PYD and CARD domain containing (PYCARD) [58] of AS-tDR000067 were enriched in actin cytoskeleton organization (ontology: biological process, G0: 0030036) and regulation of tumor necrosis factor-mediated signaling pathway (ontology: biological process, GO: 0010803), respectively (Fig. 5c). Cell proliferation-related target DEmRNAs protein tyrosine phosphatase receptor type J (PTPRJ) [59], transmembrane protein with EGF like and two follistatin like domains 2 (TMEFF2) [60], T-box transcription factor 5 (TBX5) [61], tropomyosin 1 (TPM1) [62], and Cbp/p300 
interacting transactivator with Glu/Asp rich carboxy-terminal domain 2 (CITED2) [63] of AS-tDR-009512 were enriched in negative regulation of cell migration (ontology: biological process, G0: 0030336)

(Fig. 5e). Cell proliferation-related target DEmRNAs mitofusin 2 (MFN2) [64], osteoglycin (OGN) [65], and splicing factor 1 (SF1) [66] of AS-tDR-000076 were enriched in negative regulation of smooth muscle cell proliferation (ontology: biological process, GO: 0048662) (Fig. 5g).

KEGG analysis revealed that four molecules were enriched in cell proliferation-related pathways. Cell proliferation-related target DEmRNA NUMB like endocytic adaptor protein (NUMBL) [67] of AS-tDR001370 was enriched in Notch signaling pathway (hsa04330) (Fig. 5b). Cell proliferation-related target DEmRNA protein kinase C gamma (PRKCG) [68] of AS-tDR-000067 was enriched in VEGF signaling pathway (hsa04370) (Fig. 5d). Cell proliferation-related target mRNAs coenzyme cytochrome P450 family 2 subfamily C member 8 (CYP2C8) [69] and glutathione peroxidase 7 (GPX7) [70] of AS-tDR-009512 were enriched in Arachidonic acid metabolism (hsa 00590) (Fig. 5f). Target DEmRNAs cell division cycle 42 (CDC42) [71] and PRKCG [72] of AS-tDR-000076 were enriched in VEGF signaling pathway (hsa04370) (Fig. 5h).

\section{Construction of circRNA-tsRNA interaction networks}

CircRNAs interact with miRNAs and act in the form of competing endogenous RNAs (ceRNAs) [73]. Our previous study revealed that circRNAs regulate HASMC proliferation through sponging miRNAs [74]. Based on the miRNA-like function of tsRNAs, four DEtsRNAs (AS-tDR-001370, AS-tDR-000067, AS-tDR009512, and AS-tDR-000076) were selected to construct circRNA-tsRNA interaction networks (Fig. 6). From the interaction networks, AS-tDR-001370, AS-tDR-000067, AS-tDR-009512, and AS-tDR-000076 might target 21, 71, 37, and 53 DEcircRNAs (Fig. 6).

\section{Knockdown of AS-tDR-000067 inhibits the proliferation of HASMCs}

In the AS-tDR-000067-promoter interaction network, we found an important apoptosis-related target gene p53 [75] (Fig. 2b). Through RNAhybrid [76], it was found that AS-tDR-000067 contains two binding sites on the p53 promoter (Fig. 7a). ASO was used to specifically knockdown AS-tDR-000067 in proliferative HASMCs, and the knockdown efficiency of AS-tDR-000067 was as high as 60\% (Fig. S1). Western blot confirmed that knockdown of AS-tDR-000067 promoted the expression of p53 (Fig. 7b, c). Subsequently, EdU was executed to test the proliferation rate of HASMCs, and AS-tDR-000067 suppression resulted in a reduction of up to $80 \%$ in EdU positive cells (Fig. 7d, e). Therefore, we speculate that AS-tDR-000067 may promote HASMC proliferation by targeting the p53 promoter to inhibit its transcription.

\section{Knockdown of AS-tDR-000076 inhibits the proliferation of HASMCs}

AS-tDR-000076 was selected for further analysis because GO analysis showed that the target DEmRNAs of AS-tDR-000076 were involved in negative regulation of smooth muscle cell proliferation (ontology: biological process, GO: 0048662) (Fig. 5g). Another important reason is that MFN2, an important 
proliferation inhibitor [77], is one of the target DEmRNAs of AS-tDR-000076 (Fig. 4d). Through RNAhybrid [76], it was found that the binding site of AS-tDR-000076 was located in CDS (coding sequence) and 3'UTR (untranslated region) of MFN2 (Fig. 8a). ASO was utilized to specifically knockdown AS-tDR-000076 in proliferative HASMCs, and the knockdown efficiency of AS-tDR-000076 was as high as $60 \%$ (Fig. S2). Western blot confirmed that knockdown of AS-tDR-000076 promoted the expression of MFN2 (Fig. 8b, c). Subsequently, EdU was executed to test the proliferation rat of HASMCs, and AS-tDR-000076 suppression resulted in a reduction of up to $80 \%$ in EdU positive cells (Fig. 8d, e). Therefore, we speculate that AS-tDR000076 may promote cell proliferation by targeting MFN2.

\section{Discussion}

tsRNAs, as a new type of non-coding RNA derived from tRNA, are receiving more and more attention. tsRNAs are rich in content, evolutionarily conservative, and widely present in all areas of life. These characteristics suggest that they are not the by-product of tRNA production or degradation, and they may be involved in the regulation of the body $[23,78]$. There are two hot spots in the mechanism research of tsRNAs. One is that it interacts with various proteins [54, 79-81]. The other is its miRNA-like function to inhibit the expression of target genes [23, 82]. Through the above mechanisms, tsRNAs can regulate cell proliferation [83], migration [84], apoptosis [85], and other biological processes [86]. The research focus of tsRNAs is cancer, which is involved in regulating the pathogenesis of various cancers [87], and multiple databases of tsRNAs in cancer have been constructed [88, 89]. However, research on tsRNAs is in its infancy, and its role in cardiovascular disease is still not fully understood. Huang et al. reported that miR1280 is a tsRNA derived from tRNA ${ }^{\text {Leu }}$, which binds to the $3^{\prime}-U T R$ of JAG2 and silences its expression, thereby inhibiting the proliferation, migration, and self-renewal of colorectal cancer cells mediated by the Notch signaling pathway [83]. This provides a new idea for exploring the role of tsRNAs in vascular diseases, that is, tsRNAs can regulate abnormal proliferation-related vascular diseases by inhibiting the expression of target genes.

The purpose of this paper is to screen and identify tsRNAs related to HASMC proliferation. Here, we performed high-throughput RNA sequencing and screened out 1,838 DEtsRNAs. QRT-PCR confirmed that AS-tDR-001370, AS-tDR-000067, AS-tDR-009512, and AS-tDR-000076 were up-regulated in proliferative HASMCs, and mainly located in the nucleus. Then, we predicted and screened their target genes respectively to construct tsRNA-promoter, tsRNA-mRNA, and circRNA-tsRNA interaction networks. Bioinformatics analysis showed that target genes of 4 tsRNAs (AS-tDR-001370, AS-tDR-000067, AS-tDR009512, and AS-tDR-000076) are involved in various proliferation-related terms and pathways. In tsRNApromoter interaction networks, we found that AS-tDR-001370 can target cell proliferation-related proteins CCND1 [90], SPRY2 [91], and BMPR2 [92]. The expression of CCND1 is increased in proliferative VSMCs and can promote its proliferation [90]. Thus, AS-tDR-001370 may regulate VSMC proliferation by promoting CCND1 transcription. Besides, AS-tDR-000076 can also target CCND1, indicating that AS-tDR000076 can coordinate with AS-tDR-001370 to regulate VSMC proliferation. SPRY2 can inhibit the VSMC proliferation and migration, thereby reducing neointimal growth after vascular injury [91]. Loss of 
function of BMPR2 is often found in patients with pulmonary arterial hypertension (PAH) induced by abnormal proliferation of VSMC, which has the effect of inhibiting the VSMC proliferation [92]. Thus, AStDR-001370 may promote the proliferation of VSMC by targeting SPRY2 and BMPR2 promoters and inhibiting their transcription. Cell proliferation-related proteins YAP1 [93], CDK6 [94], ATG4B [95], and p53 [48] are the target genes of AS-tDR-000067. YAP1 promotes VSMC proliferation by interacting with TEA domain transcription factor 1 (TEAD1) on the enhancer of platelet-derived growth factor receptor beta (PDGFRB) [93]. Thus, AS-tDR-000067 may regulate VSMC proliferation by regulating PDGFRB downstream pathway. CDK6, like CCND1, is an important cell cycle regulator and can promote VSMC proliferation [94]. ATG4B can promote the proliferation, invasion, migration [95], and autophagy [96] of cancer cells. Thus, AS-tDR-000067 may regulate VSMC proliferation by promoting CDK6 and ATG4B transcription. p53 is an important tumour suppressor gene, and the transcription factor it encodes is essential for the regulation of the cell cycle and apoptosis [75]. A large number of studies have confirmed that p53 can prevent atherosclerosis [97], hypertension [98], vascular stenosis [99], and other vascular remodelling diseases by inhibiting the proliferation, invasion, and migration of VSMCs and inducing their apoptosis. Our research has confirmed that knocking down AS-tDR-000067 can promote the expression of $\mathrm{p53}$, indicating that AS-tDR-000067 may promote HASMC proliferation by inhibiting the transcription of p53 (Fig. 7). However, this mechanism is not explained in this article, and further experimental verification is needed. Cell proliferation-related proteins ITPR1 [100], CALD1 [101], and RTN4 [102] are the target genes of AS-tDR-009512. ITPR1 is also called IP3R1, and its regulated $\mathrm{Ca}^{+}$signal is essential for VSMC proliferation [100]. CALD1 is related to the contractile function of VSMC [101]. RTN4 is also called Nogo, which can inhibit VSMC proliferation and migration [102]. Therefore, AS-tDR-009512 can regulate VSMC proliferation by targeting ITPR1, CALD1, and RTN4 promoters. Cell proliferation-related proteins TGFB1 [103], MAPK9 [104], and SFRP1 [105] are the target genes of AS-tDR-000076. TGFB1 can inhibit VSMC proliferation and promote its apoptosis by regulating long noncoding RNA MEG3 [103]. Bioinformatics analysis showed that MAPK9 was related to the Wnt signaling pathway, an important VSMC proliferation-related pathway [106]. Besides, SFRP1 was an important inhibitor of the Wnt signaling pathway, suggesting that AS-tDR-000076 may regulate VSMC proliferation through it [107].

In tsRNA-mRNA interaction networks, AS-tDR-001370's target mRNA NUMBL is involved in the inhibition of the Notch signaling pathway [67, 108], an important VSMC proliferation-related pathway [109]. It is suggested that AS-tDR-001370 can promote VSMC proliferation by reducing the effect of NUMBL. Also, the target genes PHB2 [58] and CLMN [59] of AS-tDR-001370 can inhibit cell proliferation, suggesting AStDR-001370 may also promote proliferation through these two ways. AS-tDR-000067's target mRNA PYCARD is a pro-apoptotic molecule [54]. Thus, AS-tDR-000067 may inhibit the process of apoptosis by targeting PYCARD. As the downstream target genes of AS-tDR-009512, TPM1 [110], TMEFF2 [111], and PTPRJ $[112,113]$ are involved in cell proliferation and metastasis. It has been reported that TPM1 is involved in the inhibition of VSMC proliferation and metastasis [110]. MicroRNA-21 regulates VSMC function of lower extremity arteriosclerosis obliterans by targeting TPM1 [62]. Therefore, AS-tDR-009512 may drive VSMC proliferation by downregulating TPM1. As a target of many molecules, TMEFF2 participates in the inhibition of tumour cell proliferation, migration, and invasion by inhibiting the MAPK 
signaling pathway [111]. The involvement of the MAPK signaling pathway in VSMC proliferation has been confirmed [114]. Here, we propose that AS-tDR-009512 participates in VSMC proliferation through the MAPK signaling pathway. Similarly, PTPRJ (also known as DEP-1 or CD148) can inhibit the proliferation and migration of multiple cells through the ERK [112] or PI3K signaling pathways [113] and is closely related to cytoskeletal rearrangements [59]. TMEFF2 and PTPRJ also serve as target genes of AS-tDR-000076, indicating that AS-tDR-009512 and AS-tDR-000076 may coordinate the proliferation of VSMC through the MAPK and PI3K signaling pathways. MFN2 [77], SF1 [66, 115], and OGN [65, 116] are target genes of AS-tDR-000076, which are down-regulated in proliferative HASMCs. It has been reported that MFN2 can inhibit the proliferation and promote the apoptosis of VSMCs by inhibiting the Ras-RafERK1/2 pathway [77] and PI3K-Akt pathway [117], respectively. Therefore, AS-tDR-000076 may promote the VSMC proliferation by inhibiting the MFN2-Ras-Raf-ERK1/2 pathway. Wnt signaling pathway is an important proliferation-related pathway, which plays an important role in the VSMC proliferation [118]. SF1 is a downstream molecule of the Wnt signaling pathway [119] and can inhibit the VSMC proliferation $[66,115]$. Hence, AS-tDR-000076 may regulate the VSMC proliferation by silencing SF1. Similarly, OGN is also involved in the inhibition of proliferation. It can negatively regulate the epidermal growth factor receptor (EGFR) signaling pathway [116] and vascular endothelial growth factor receptor 2 (VEGF2) signaling pathway [65], but there is no study on its regulation of VSMC proliferation. It has been reported that OGN inhibits the proliferation and migration of cancer cells through the PI3K/Akt/mTOR signaling pathway [120]. Therefore, whether AS-tDR-000076 also regulates the VSMC proliferation through ONG/PI3K/Akt/mTOR signaling pathway is also worthy of further discussion. In addition to the VSMC proliferation, AS-tDR-000076 is also involved in the regulation of cardiomyocyte function related molecules, such as the transcription factor Nkx2.5 that regulates cardiomyocyte contraction [121], EH domain containing 3 (EHD3) that maintains the excitability and physiological function of myocardial cell membranes [122], cardiac conduction related SCN10A (sodium voltage-gated channel alpha subunit 10) [123], their insufficient expression are involved in the occurrence of diseases such as abnormal cardiac conduction, ventricular fibrillation, and heart failure. Therefore, further research on the function of AS-tDR000076 is helpful to understand the mechanism of heart disease and may provide a new therapeutic target.

The purpose of this article is to screen the tsRNAs involved in the HASMC proliferation and explore their miRNA-like functions. The tsRNA-promoter interaction networks show that AS-tDR-000067 can regulate more than 800 target genes, of which p53 is involved in VSMC proliferation [48]. EdU fluorescent stain and western blot confirmed that AS-tDR-000067 may promote HASMC proliferation by targeting p53 promoter. The tsRNA-mRNA interaction networks show that AS-tDR-000076 can regulate more than 300 target genes, of which MFN2 is involved in VSMC proliferation [77]. Studies have reported that miRNA can promote cell proliferation by down-regulating MFN2, such as MiR-93 [124] and MicroRNA-497 [125].

Therefore, we speculate that AS-tDR-000076 promotes proliferation by inhibiting the expression of MFN2. EdU fluorescent stain and western blot confirmed that AS-tDR-000076 may promote HASMC proliferation by targeting MFN2. However, the specific regulatory mechanism of AS-tDR-000067 and AS-tDR-000076 on target gene was not covered in this article, and experiments are needed to further explore. 


\section{Conclusions}

In this article, we conducted a comprehensive analysis of the tsRNA expression profiles of proliferative and quiescent HASMCs. Subsequently, qRT-PCR was performed to confirm the sequencing results. The expression of AS-tDR-001370, AS-tDR-000067, AS-tDR-009512, and AS-tDR-000076 was up-regulated in proliferative HASMCs and mainly located in the cytoplasm. We constructed their tsRNA-promoter, tsRNAmRNA, and circRNA-tsRNA interaction networks. DAVID database was used to analyze the function of target genes, and pointed out that AS-tDR-001370, AS-tDR-000067, AS-tDR-009512, and AS-tDR-000076 may positively regulate VSMC proliferation. Finally, it was confirmed that AS-tDR-000067 and AS-tDR000076 may participate in the proliferation of HASMC through down-regulation of p53 and MFN2, respectively, indicating that they may become new targets for the treatment of vascular diseases. This paper reports the role of tsRNAs in human vascular disease for the first time, emphasizing the extensive regulation of tsRNAs, and providing new insights into the mechanism of vascular disease.

\section{Abbreviations}




\begin{tabular}{|c|c|}
\hline VSMC & vascular smooth muscle cell \\
\hline tsRNA & tRNA-derived small RNA \\
\hline HASMC & human aortic smooth muscle cell \\
\hline qRT-PCR & quantitative real-time PCR \\
\hline circRNA & circular RNA \\
\hline GO & Gene Ontology \\
\hline KEGG & Kyoto Encyclopedia of Genes and Genomes \\
\hline MFN2 & mitofusin 2 \\
\hline CCA & rat common carotid artery \\
\hline FAS & FAS cell surface death receptor \\
\hline miRNA & microRNA \\
\hline YBX1 & Y-box binding protein 1 \\
\hline 5'-SHOT-RNA & 5'-sex hormone-dependent tRNA-derived RNA \\
\hline DEtsRNA & differentially expressed tsRNA \\
\hline SMCM & smooth muscle cell medium \\
\hline FBS & fetal bovine serum \\
\hline SMCGS & smooth muscle cell growth supplement \\
\hline smRNA & small RNA \\
\hline DEmRNA & differentially expressed mRNA \\
\hline DEcircRNA & differentially expressed circRNA \\
\hline ASO & antisense oligonucleotide \\
\hline NC & negative control \\
\hline PVDF & polyvinylidene fluoride \\
\hline TBS & Tris-buffered saline \\
\hline EdU & 5-ethynyl-2'-deoxyuridine \\
\hline SD & standard deviation \\
\hline AGO & Argonaute \\
\hline CCND1 & cyclin D1 \\
\hline SPRY2 & sprouty RTK signaling antagonist 2 \\
\hline
\end{tabular}




\begin{tabular}{|c|c|}
\hline VSMC & vascular smooth muscle cell \\
\hline BMPR2 & bone morphogenetic protein receptor type 2 \\
\hline YAP1 & Yes1 associated transcriptional regulator \\
\hline HMGA2 & high mobility group AT-hook 2 \\
\hline CDK6 & cyclin dependent kinase 6 \\
\hline ATG4B & autophagy related $4 \mathrm{~B}$ cysteine peptidase \\
\hline NR2F1 & nuclear receptor subfamily 2 group F member 1 \\
\hline AGGF1 & angiogenic factor with G-patch and FHA domains 1 \\
\hline CALD1 & caldesmon 1 \\
\hline RTN4 & reticulon 4 \\
\hline SFRP1 & secreted frizzled related protein 1 \\
\hline TGFB1 & transforming growth factor beta 1 \\
\hline RELA & RELA proto-oncogene \\
\hline FGF9 & fibroblast growth factor 9 \\
\hline CACNA1G & calcium voltage-gated channel subunit alpha $1 \mathrm{G}$ \\
\hline CCND3 & cyclin D3 \\
\hline CDK2 & cyclin dependent kinase 2 \\
\hline CAMK2D & calcium/calmodulin dependent protein kinase II delta \\
\hline CCND2 & cyclin D2 \\
\hline MAPK9 & mitogen-activated protein kinase 9 \\
\hline PHB2 & prohibitin 2 \\
\hline ATXN3 & ataxin 3 \\
\hline PYCARD & PYD and CARD domain containing \\
\hline PTPRJ & protein tyrosine phosphatase receptor type $\mathrm{J}$ \\
\hline TMEFF2 & transmembrane protein with EGF like and two follistatin like domains 2 \\
\hline TBX5 & T-box transcription factor 5 \\
\hline TPM1 & tropomyosin 1 \\
\hline CITED2 & Cbp/p300 interacting transactivator with Glu/Asp rich carboxy-terminal domain 2 \\
\hline OGN & osteoglycin \\
\hline
\end{tabular}




\begin{tabular}{|ll|}
\hline VSMC & vascular smooth muscle cell \\
\hline SF1 & splicing factor 1 \\
\hline NUMBL & NUMB like endocytic adaptor protein \\
\hline PRKCG & protein kinase C gamma \\
\hline CYP2C8 & coenzyme cytochrome P450 family 2 subfamily C member 8 \\
\hline GPX7 & glutathione peroxidase 7 \\
\hline CDC42 & cell division cycle 42 \\
\hline CDS & coding sequence \\
\hline 3'-UTR & untranslated region \\
\hline PAH & pulmonary arterial hypertension \\
\hline TEAD1 & TEA domain transcription factor 1 \\
\hline PDGFRB & platelet-derived growth factor receptor beta \\
\hline EHD3 & EH domain containing 3 \\
\hline SCN10A & sodium voltage-gated channel alpha subunit 10 \\
\hline
\end{tabular}

\section{Declarations}

\section{Ethics approval and consent to participate}

Not applicable

\section{Consent for publication}

Informed consent was obtained from all individual participants included in the study.

\section{Availability of data and materials}

The original data discussed in this study have been deposited in NCBI Gene Expression Omnibus and are accessible with the GEO Series accession number GSE164540

(https://www.ncbi.nlm.nih.gov/geo/query/acc.cgi?acc=GSE164540).

\section{Competing interests}

The authors declare no competing interests.

\section{Funding}


This study was funded by the National Natural Science Foundation of China (81670273 and 81200215) and the Natural Science Foundation of Hebei Province (H2019206150).

\section{Authors' contributions}

S.G.S. and B.L. designed and supervised the study. J.Z.Z., J.J.L., M.G., Y.W., M.Y.D, H.H.L., B.S., P.K., and K.L. performed the bioinformatics analysis. J.Z.Z., J.J.L., M.G., K.X.L, L.Y.Y., and S.F.W. performed the experiments. J.Z.Z., J.J.L., and M.G. wrote the manuscript. All authors read and approved the final manuscript.

\section{Acknowledgements}

Not applicable

\section{Authors' information}

*Correspondence: Shao-Guang Sun (email: sunshaoguang00@163.com) and Bin Li (email: libin_cj@163.com)

† Jian-Zhi Zhao, Jia-Jie Lin and Miao Gong contribute equally to this work

Department of Biochemistry and Molecular Biology, Key Laboratory of Medical Biotechnology of Hebei Province, Cardiovascular Medical Science Center, Hebei Medical University, Shijiazhuang 050017, China.

\section{References}

1. Collaborators GBDCoD. Global, regional, and national age-sex specific mortality for 264 causes of death, 1980-2016: a systematic analysis for the Global Burden of Disease Study 2016. Lancet. 2017;390:1151-210.

2. Owens GK, Kumar MS, Wamhoff BR. Molecular regulation of vascular smooth muscle cell differentiation in development and disease. Physiol Rev. 2004;84:767-801.

3. Wang XP, Zhang W, Liu XQ, Wang WK, Yan F, Dong WQ, et al. Arginase I enhances atherosclerotic plaque stabilization by inhibiting inflammation and promoting smooth muscle cell proliferation. Eur Heart J. 2014;35:911-9.

4. Fan Y, Gu X, Zhang J, Sinn K, Klepetko W, Wu N, et al.: TWIST1 Drives Smooth Muscle Cell Proliferation in Pulmonary Hypertension via Loss of GATA-6 and BMPR2. Am J Respir Crit Care Med. (2020).

5. Jeong K, Kim JH, Murphy JM, Park H, Kim SJ, Rodriguez YAR, et al. Nuclear Focal Adhesion Kinase Controls Vascular Smooth Muscle Cell Proliferation and Neointimal Hyperplasia Through GATA4Mediated Cyclin D1 Transcription. Circ Res. 2019;125:152-66.

6. Matsuoka T, Wada J, Hashimoto I, Zhang Y, Eguchi J, Ogawa N, et al. Gene delivery of Tim44 reduces mitochondrial superoxide production and ameliorates neointimal proliferation of injured carotid 
artery in diabetic rats. Diabetes. 2005;54:2882-90.

7. Lee YS, Shibata Y, Malhotra A, Dutta A. A novel class of small RNAs: tRNA-derived RNA fragments (tRFs). Genes development. 2009;23:2639-49.

8. Li Q, Hu B, Hu GW, Chen CY, Niu X, Liu J, et al. tRNA-Derived Small Non-Coding RNAs in Response to Ischemia Inhibit Angiogenesis. Sci Rep. 2016;6:20850.

9. Maute RL, Schneider C, Sumazin P, Holmes A, Califano A, Basso K, et al. tRNA-derived microRNA modulates proliferation and the DNA damage response and is down-regulated in B cell lymphoma. Proc Natl Acad Sci U S A. 2013;110:1404-9.

10. Goodarzi H, Liu X, Nguyen HC, Zhang S, Fish L, Tavazoie SF. Endogenous tRNA-Derived Fragments Suppress Breast Cancer Progression via YBX1 Displacement. Cell. 2015;161:790-802.

11. Honda S, Loher P, Shigematsu M, Palazzo JP, Suzuki R, Imoto I, et al. Sex hormone-dependent tRNA halves enhance cell proliferation in breast and prostate cancers. Proc Natl Acad Sci U S A. 2015;112:E3816-25.

12. Balatti V, Nigita G, Veneziano D, Drusco A, Stein GS, Messier TL, et al. tsRNA signatures in cancer. Proc Natl Acad Sci U S A. 2017;114:8071-6.

13. Zhang M, Li F, Wang J, He W, Li Y, Li H, et al. tRNA-derived fragment tRF-03357 promotes cell proliferation, migration and invasion in high-grade serous ovarian cancer. Onco Targets Ther. 2019;12:6371-83.

14. Zhu XL, Li T, Cao Y, Yao QP, Liu X, Li Y, et al. tRNA-derived fragments tRF(GInCTG) induced by arterial injury promote vascular smooth muscle cell proliferation. Mol Ther Nucleic Acids. 2021;23:603-13.

15. Farina NH, Scalia S, Adams CE, Hong D, Fritz AJ, Messier TL, et al. Identification of tRNA-derived small RNA (tsRNA) responsive to the tumor suppressor, RUNX1, in breast cancer. J Cell Physiol. 2020;235:5318-27.

16. Zhong F, Hu Z, Jiang K, Lei B, Wu Z, Yuan G, et al. Complement C3 activation regulates the production of tRNA-derived fragments Gly-tRFs and promotes alcohol-induced liver injury and steatosis. Cell Research. 2019;29:548-61.

17. Schorn AJ, Gutbrod MJ, LeBlanc C, Martienssen R. LTR-Retrotransposon Control by tRNA-Derived Small RNAs. Cell. 170, 61-71 e11 (2017).

18. Ivanov P, O'Day E, Emara MM, Wagner G, Lieberman J, Anderson P. G-quadruplex structures contribute to the neuroprotective effects of angiogenin-induced tRNA fragments. Proc Natl Acad Sci U S A. 2014;111:18201-6.

19. Kruger J, Rehmsmeier M. RNAhybrid: microRNA target prediction easy, fast and flexible. Nucleic Acids Res. 2006;34:W451-4.

20. John B, Enright AJ, Aravin A, Tuschl T, Sander C, Marks DS. Human MicroRNA targets. PLoS Biol. 2004;2:e363.

21. Agarwal V, Bell GW, Nam JW, Bartel DP. Predicting effective microRNA target sites in mammalian mRNAs. Elife. 2015;4:e05005. 
22. Huang da W, Sherman BT, Lempicki RA. Systematic and integrative analysis of large gene lists using DAVID bioinformatics resources. Nat Protoc. 2009;4:44-57.

23. Kumar P, Anaya J, Mudunuri SB, Dutta A. Meta-analysis of tRNA derived RNA fragments reveals that they are evolutionarily conserved and associate with AGO proteins to recognize specific RNA targets. $12,78$.

24. Li H, Fan J, Zhao Y, Zhang X, Dai B, Zhan J, et al. Nuclear miR-320 Mediates Diabetes-Induced Cardiac Dysfunction by Activating Transcription of Fatty Acid Metabolic Genes to Cause Lipotoxicity in the Heart. Circ Res. 2019;125:1106-20.

25. Chaluvally-Raghavan P, Jeong KJ, Pradeep S, Silva AM, Yu S, Liu W, et al. Direct Upregulation of STAT3 by MicroRNA-551b-3p Deregulates Growth and Metastasis of Ovarian Cancer. Cell Rep. 2016;15:1493-504.

26. Qu H, Zheng L, Pu J, Mei H, Xiang X, Zhao X, et al. miRNA-558 promotes tumorigenesis and aggressiveness of neuroblastoma cells through activating the transcription of heparanase. Hum Mol Genet. 2015;24:2539-51.

27. Younger ST, Corey DR. Transcriptional gene silencing in mammalian cells by miRNA mimics that target gene promoters. Nucleic Acids Res. 2011;39:5682-91.

28. Li Z, Lan X, Han R, Wang J, Huang Y, Sun J, et al. miR-2478 inhibits TGFbeta1 expression by targeting the transcriptional activation region downstream of the TGFbeta1 promoter in dairy goats. Sci Rep. 2017;7:42627.

29. Di Mauro V, Crasto S, Colombo FS, Di Pasquale E, Catalucci D. Wnt signalling mediates miR-133a nuclear re-localization for the transcriptional control of Dnmt3b in cardiac cells. Sci Rep. 2019;9:9320.

30. Heiss EH, Liu R, Waltenberger B, Khan S, Schachner D, Kollmann P, et al. Plumericin inhibits proliferation of vascular smooth muscle cells by blocking STAT3 signaling via S-glutathionylation. Sci Rep. 2016;6:20771.

31. Zhang C, Chaturvedi D, Jaggar L, Magnuson D, Lee JM, Patel TB. Regulation of vascular smooth muscle cell proliferation and migration by human sprouty 2. Arterioscler Thromb Vasc Biol. 2005;25:533-8.

32. Fan Y, Gu X, Zhang J, Sinn K, Klepetko W, Wu N, et al. TWIST1 Drives Smooth Muscle Cell Proliferation in Pulmonary Hypertension via Loss of GATA-6 and BMPR2. Am J Respir Crit Care Med. 2020;202:1283-96.

33. Yu Q, Li W, Jin R, Yu S, Xie D, Zheng X, et al. PI3Kgamma (Phosphoinositide 3-Kinase gamma) Regulates Vascular Smooth Muscle Cell Phenotypic Modulation and Neointimal Formation Through CREB (Cyclic AMP-Response Element Binding Protein)/YAP (Yes-Associated Protein) Signaling. Arterioscler Thromb Vasc Biol. 2019;39:e91-105.

34. Wu X, Serna VA, Thomas J, Qiang W, Blumenfeld ML, Kurita T. Subtype-Specific Tumor-Associated Fibroblasts Contribute to the Pathogenesis of Uterine Leiomyoma. Cancer Res. 2017;77:6891-901. 
35. Zhang J, Deng B, Jiang X, Cai M, Liu N, Zhang S, et al. All-Trans-Retinoic Acid Suppresses Neointimal Hyperplasia and Inhibits Vascular Smooth Muscle Cell Proliferation and Migration via Activation of AMPK Signaling Pathway. Front Pharmacol. 2019;10:485.

36. Liu PF, Chen HC, Cheng JS, Tsai WL, Lee HP, Wang SC, et al.: Association of ATG4B and Phosphorylated ATG4B Proteins with Tumorigenesis and Prognosis in Oral Squamous Cell Carcinoma. Cancers (Basel). 11, (2019).

37. Lim YH, Ryu J, Kook H, Kim YK. Identification of Long Noncoding RNAs Involved in Differentiation and Survival of Vascular Smooth Muscle Cells. Mol Ther Nucleic Acids. 2020;22:209-21.

38. Luo Y, Huang C. CircSFMBT2 facilitates vascular smooth muscle cell proliferation by targeting miR331-3p/HDAC5. Life Sci. 2021;264:118691.

39. Goikuria H, Freijo MDM, Vega Manrique R, Sastre M, Elizagaray E, Lorenzo A, et al.: Characterization of Carotid Smooth Muscle Cells during Phenotypic Transition. Cells. 7, (2018).

40. Chick HE, Nowrouzi A, Fronza R, McDonald RA, Kane NM, Alba R, et al. Integrase-deficient lentiviral vectors mediate efficient gene transfer to human vascular smooth muscle cells with minimal genotoxic risk. Hum Gene Ther. 2012;23:1247-57.

41. Tang Z, Chen W, Xu Y, Lin X, Liu X, Li Y, et al. miR-4721, Induced by EBV-miR-BART22, Targets GSK3beta to Enhance the Tumorigenic Capacity of NPC through the WNT/beta-catenin Pathway. Mol Ther Nucleic Acids. 2020;22:557-71.

42. Lin XH, Liu HH, Hsu SJ, Zhang R, Chen J, Chen J, et al. Norepinephrine-stimulated HSCs secrete sFRP1 to promote HCC progression following chronic stress via augmentation of a Wnt16B/betacatenin positive feedback loop. J Exp Clin Cancer Res. 2020;39:64.

43. de Streel G, Bertrand C, Chalon N, Lienart S, Bricard O, Lecomte S, et al. Selective inhibition of TGFbeta1 produced by GARP-expressing Tregs overcomes resistance to PD-1/PD-L1 blockade in cancer. Nat Commun. 2020;11:4545.

44. Kong P, Yu Y, Wang L, Dou YQ, Zhang XH, Cui Y, et al. circ-Sirt1 controls NF-kappaB activation via sequence-specific interaction and enhancement of SIRT1 expression by binding to miR-132/212 in vascular smooth muscle cells. Nucleic Acids Res. 2019;47:3580-93.

45. Li A, Jia P, Mallik S, Fei R, Yoshioka H, Suzuki A, et al. Critical microRNAs and regulatory motifs in cleft palate identified by a conserved miRNA-TF-gene network approach in humans and mice. Brief Bioinform. 2020;21:1465-78.

46. Zhang B, Chen L, Bai YG, Song JB, Cheng JH, Ma HZ, et al. miR-137 and its target T-type CaV 3.1 channel modulate dedifferentiation and proliferation of cerebrovascular smooth muscle cells in simulated microgravity rats by regulating calcineurin/NFAT pathway. Cell Prolif. 2020;53:e12774.

47. Liang KW, Yin SC, Ting CT, Lin SJ, Hsueh CM, Chen CY, et al. Berberine inhibits platelet-derived growth factor-induced growth and migration partly through an AMPK-dependent pathway in vascular smooth muscle cells. Eur J Pharmacol. 2008;590:343-54.

48. Wang H, He F, Liang B, Jing Y, Zhang P, Liu W, et al.: p53-Dependent LincRNA-p21 Protects Against Proliferation and Anti-apoptosis of Vascular Smooth Muscle Cells in Atherosclerosis by Upregulating 
SIRT7 via MicroRNA-17-5p. J Cardiovasc Transl Res. (2020).

49. Tanner FC, Boehm M, Akyurek LM, San H, Yang ZY, Tashiro J, et al. Differential effects of the cyclindependent kinase inhibitors p27(Kip1), p21(Cip1), and p16(Ink4) on vascular smooth muscle cell proliferation. Circulation. 2000;101:2022-5.

50. Afroze T, Sadi AM, Momen MA, Gu S, Heximer S, Husain M. c-Myb-dependent inositol 1,4,5trisphosphate receptor type-1 expression in vascular smooth muscle cells. Arterioscler Thromb Vasc Biol. 2007;27:1305-11.

51. Wang H, Jin Z, Pei T, Song W, Gong Y, Chen D, et al. Long noncoding RNAs C2dat1 enhances vascular smooth muscle cell proliferation and migration by targeting MiR-34a-5p. J Cell Biochem. 2019;120:3001-8.

52. Wang C, Zhang L, Ke L, Ding W, Jiang S, Li D, et al. Primary effusion lymphoma enhancer connectome links super-enhancers to dependency factors. Nat Commun. 2020;11:6318.

53. Liu J, Shu B, Zhou Z, Xu Y, Liu Y, Wang P, et al. Involvement of miRNA203 in the proliferation of epidermal stem cells during the process of DM chronic wound healing through Wnt signal pathways. Stem Cell Res Ther. 2020;11:348.

54. Lalande S, Merret R, Salinas-Giege T, Drouard L. Arabidopsis tRNA-derived fragments as potential modulators of translation. RNA Biol. 2020;17:1137-48.

55. Deng J, Ptashkin RN, Chen Y, Cheng Z, Liu G, Phan T, et al. Respiratory Syncytial Virus Utilizes a tRNA Fragment to Suppress Antiviral Responses Through a Novel Targeting Mechanism. Mol Ther. 2015;23:1622-9.

56. Zhang L, Niu H, Ma J, Yuan BY, Chen YH, Zhuang Y, et al. The molecular mechanism of LncRNA34amediated regulation of bone metastasis in hepatocellular carcinoma. Mol Cancer. 2019;18:120.

57. Neves-Carvalho A, Logarinho E, Freitas A, Duarte-Silva S, Costa Mdo C, Silva-Fernandes A, et al. Dominant negative effect of polyglutamine expansion perturbs normal function of ataxin-3 in neuronal cells. Hum Mol Genet. 2015;24:100-17.

58. Miao H, Wang L, Zhan H, Dai J, Chang Y, Wu F, et al. A long noncoding RNA distributed in both nucleus and cytoplasm operates in the PYCARD-regulated apoptosis by coordinating the epigenetic and translational regulation. PLoS Genet. 2019;15:e1008144.

59. Balavenkatraman KK, Jandt E, Friedrich K, Kautenburger T, Pool-Zobel BL, Ostman A, et al. DEP-1 protein tyrosine phosphatase inhibits proliferation and migration of colon carcinoma cells and is upregulated by protective nutrients. Oncogene. 2006;25:6319-24.

60. Li K, Han H, Gu W, Cao C, Zheng P. Long non-coding RNA LINC01963 inhibits progression of pancreatic carcinoma by targeting miR-641/TMEFF2. Biomed Pharmacother. 2020;129:110346.

61. Kim J, Siverly AN, Chen D, Wang M, Yuan Y, Wang Y, et al. Ablation of miR-10b Suppresses OncogeneInduced Mammary Tumorigenesis and Metastasis and Reactivates Tumor-Suppressive Pathways. Cancer Res. 2016;76:6424-35.

62. Wang M, Li W, Chang GQ, Ye CS, Ou JS, Li XX, et al. MicroRNA-21 regulates vascular smooth muscle cell function via targeting tropomyosin 1 in arteriosclerosis obliterans of lower extremities. 
Arterioscler Thromb Vasc Biol. 2011;31:2044-53.

63. Shin SH, Lee GY, Lee M, Kang J, Shin HW, Chun YS, et al. Aberrant expression of CITED2 promotes prostate cancer metastasis by activating the nucleolin-AKT pathway. Nat Commun. 2018;9:4113.

64. Sorianello E, Soriano FX, Fernandez-Pascual S, Sancho A, Naon D, Vila-Caballer M, et al. The promoter activity of human Mfn2 depends on Sp1 in vascular smooth muscle cells. Cardiovasc Res. 2012;94:38-47.

65. Wu QH, Ma Y, Ruan CC, Yang Y, Liu XH, Ge Q, et al. Loss of osteoglycin promotes angiogenesis in limb ischaemia mouse models via modulation of vascular endothelial growth factor and vascular endothelial growth factor receptor 2 signalling pathway. Cardiovasc Res. 2017;113:70-80.

66. Cattaruzza M, Nogoy N, Wojtowicz A, Hecker M. Zinc finger motif-1 antagonizes PDGF-BB-induced growth and dedifferentiation of vascular smooth muscle cells. FASEB J. 2012;26:4864-75.

67. Swarnkar G, Karuppaiah K, Mbalaviele G, Chen TH, Abu-Amer Y. Osteopetrosis in TAK1-deficient mice owing to defective NF-kappaB and NOTCH signaling. Proc Natl Acad Sci U S A. 2015;112:154-9.

68. Verbeek DS, Goedhart J, Bruinsma L, Sinke RJ, Reits EA. PKC gamma mutations in spinocerebellar ataxia type 14 affect $\mathrm{C} 1$ domain accessibility and kinase activity leading to aberrant MAPK signaling. J Cell Sci. 2008;121:2339-49.

69. Li K, Chen Y. CYP2C8 regulated by GAS5/miR-382-3p exerts anti-cancerous properties in liver cancer. Cancer Biol Ther. 2020;21:1145-53.

70. Peng D, Hu T, Soutto M, Belkhiri A, Zaika A, El-Rifai W. Glutathione peroxidase 7 has potential tumour suppressor functions that are silenced by location-specific methylation in oesophageal adenocarcinoma. Gut. 2014;63:540-51.

71. Andre-Gregoire G, Dilasser F, Chesne J, Braza F, Magnan A, Loirand G, et al: Targeting of Rac1 prevents bronchoconstriction and airway hyperresponsiveness. J Allergy Clin Immunol. 142, 824833 e823 (2018).

72. Liang F, Li L, Wang M, Niu X, Zhan J, He X, et al. Molecular network and chemical fragment-based characteristics of medicinal herbs with cold and hot properties from Chinese medicine. $J$ Ethnopharmacol. 2013;148:770-9.

73. Hong X, Liu N, Liang Y, He Q, Yang X, Lei Y, et al. Circular RNA CRIM1 functions as a ceRNA to promote nasopharyngeal carcinoma metastasis and docetaxel chemoresistance through upregulating FOXQ1. Mol Cancer. 2020;19:33.

74. Chen W, Lin J, Li B, Cao S, Li H, Zhao J, et al. Screening and functional prediction of differentially expressed circRNAs in proliferative human aortic smooth muscle cells. J Cell Mol Med. 2020;24:4762-72.

75. Muller PA, Vousden KH. Mutant p53 in cancer: new functions and therapeutic opportunities. Cancer Cell. 2014;25:304-17.

76. Krüger J, Rehmsmeier M. RNAhybrid: microRNA target prediction easy, fast and flexible. Nucleic Acids Res. 2006;34:W451-4. 
77. Chen KH, Guo X, Ma D, Guo Y, Li Q, Yang D, et al. Dysregulation of HSG triggers vascular proliferative disorders. Nat Cell Biol. 2004;6:872-83.

78. Soares AR, Santos M. Discovery and function of transfer RNA-derived fragments and their role in disease. Wiley Interdiscip Rev RNA. 8, (2017).

79. Guzzi N, Ciesla M, Ngoc PCT, Lang S, Arora S, Dimitriou M, et al. Pseudouridylation of tRNA-Derived Fragments Steers Translational Control in Stem Cells. Cell. 2018;173:1204-16.e1226.

80. Kim HK, Xu J, Chu K, Park H, Jang H, Li P, et al: A tRNA-Derived Small RNA Regulates Ribosomal Protein S28 Protein Levels after Translation Initiation in Humans and Mice. Cell Rep. 29, 3816-3824 e3814 (2019).

81. Sobala A, Hutvagner G. Small RNAs derived from the $5^{\prime}$ end of tRNA can inhibit protein translation in human cells. RNA Biol. 2013;10:553-63.

82. Martinez G, Choudury SG, Slotkin RK. tRNA-derived small RNAs target transposable element transcripts. Nucleic Acids Res. 2017;45:5142-52.

83. Huang B, Yang H, Cheng X, Wang D, Fu S, Shen W, et al. tRF/miR-1280 Suppresses Stem Cell-like Cells and Metastasis in Colorectal Cancer. Cancer Res. 2017;77:3194-206.

84. Falconi M, Giangrossi M, Zabaleta ME, Wang J, Gambini V, Tilio M, et al. A novel 3'-tRNA(Glu)-derived fragment acts as a tumor suppressor in breast cancer by targeting nucleolin. FASEB J. 2019;33:13228-40.

85. Saikia M, Jobava R, Parisien M, Putnam A, Krokowski D, Gao XH, et al. Angiogenin-cleaved tRNA halves interact with cytochrome $\mathrm{c}$, protecting cells from apoptosis during osmotic stress. Mol Cell Biol. 2014;34:2450-63.

86. Krishna S, Yim DG, Lakshmanan V, Tirumalai V, Koh JL, Park JE, et al. Dynamic expression of tRNAderived small RNAs define cellular states. EMBO Rep. 2019;20:e47789.

87. Zhu L, Ge J, Li T, Shen Y, Guo J. tRNA-derived fragments and tRNA halves: The new players in cancers. Cancer Lett. 2019;452:31-7.

88. Yao D, Sun X, Zhou L, Amanullah M, Pan X, Liu Y, et al. OncotRF: an online resource for exploration of tRNA-derived fragments in human cancers. RNA Biol. 2020;17:1081-91.

89. Pliatsika V, Loher P, Magee R, Telonis AG, Londin E, Shigematsu M, et al. MINTbase v2.0: a comprehensive database for tRNA-derived fragments that includes nuclear and mitochondrial fragments from all The Cancer Genome Atlas projects. Nucleic Acids Res. 2018;46:D152-9.

90. Xiao QZ, Zhang F, Grassia G, Hu YH, Zhang ZY, Xing QR, et al. Matrix Metalloproteinase-8 Promotes Vascular Smooth Muscle Cell Proliferation and Neointima Formation. Arterioscl Throm Vas. 2014;34:90-+.

91. Zhang CX, Chaturvedi D, Jaggar L, Magnuson D, Lee JM, Patel TB. Regulation of vascular smooth muscle cell proliferation and migration by human sprouty 2. Arterioscl Throm Vas. 2005;25:533-8.

92. Luo T, Cui SJ, Bian CJ, Yu XC. Crosstalk between TGF-beta/Smad3 and BMP/BMPR2 signaling pathways via miR-17-92 cluster in carotid artery restenosis. Mol Cell Biochem. 2014;389:169-76. 
93. Osman I, Yu LY, Kang XH, Ahmed A, Hu GQ, Zhang W, et al.: Yes-associated Protein Upregulates Platelet Derived Growth Factor Receptor Beta to Promote Vascular Smooth Muscle Cell Proliferation and Neointima Formation. Arterioscl Throm Vas. 39, (2019).

94. Zhang JZ, Deng B, Jiang XL, Cai M, Liu NN, Zhang SW, et al.: All-Trans-Retinoic Acid Suppresses Neointimal Hyperplasia and Inhibits Vascular Smooth Muscle Cell Proliferation and Migration via Activation of AMPK Signaling Pathway. Frontiers in Pharmacology. 10, (2019).

95. Liu PF, Chen HC, Cheng JS, Tsai WL, Lee HP, Wang SC, et al.: Association of ATG4B and Phosphorylated ATG4B Proteins with Tumorigenesis and Prognosis in Oral Squamous Cell Carcinoma. Cancers. 11, (2019).

96. Fu YY, Hong L, Xu JC, Zhong GP, Gu Q, Gu QQ, et al. Discovery of a small molecule targeting autophagy via ATG4B inhibition and cell death of colorectal cancer cells in vitro and in vivo. Autophagy. 2019;15:295-311.

97. Mercer J, Figg N, Stoneman V, Braganza D, Bennett MR. Endogenous p53 protects vascular smooth muscle cells from apoptosis and reduces atherosclerosis in ApoE knockout mice. Circ Res. 2005;96:667-74.

98. Zehendner CM, Valasarajan C, Werner A, Boeckel JN, Bischoff FC, John D, et al. Long Noncoding RNA TYKRIL Plays a Role in Pulmonary Hypertension via the p53-mediated Regulation of PDGFRbeta. Am J Respir Crit Care Med. 2020;202:1445-57.

99. Forte A, Finicelli M, Grossi M, Vicchio M, Alessio N, Sante P, et al. DNA damage and repair in a model of rat vascular injury. Clin Sci (Lond). 2010;118:473-85.

100. Afroze T, Sadi A, Momen MA, Gu S, Heximer S, Husain M. c-Myb-dependent inositol 1,4,5trisphosphate receptor type-1 expression in vascular smooth muscle cells. Arterioscl Throm Vas. 2007;27:1305-11.

101. Goikuria H, Freijo MD, Manrique RV, Sastre M, Elizagaray E, Lorenzo A, et al.: Characterization of Carotid Smooth Muscle Cells during Phenotypic Transition. Cells. 7, (2018).

102. Chick HE, Nowrouzi A, Fronza R, McDonald RA, Kane NM, Alba R, et al. Integrase-Deficient Lentiviral Vectors Mediate Efficient Gene Transfer to Human Vascular Smooth Muscle Cells with Minimal Genotoxic Risk. Human Gene Therapy. 2012;23:1247-57.

103. Liu WF, Luo ML, Zou L, Liu XX, Wang RL, Tao H, et al. uNK cell-derived TGF-beta 1 regulates the long noncoding RNA MEG3 to control vascular smooth muscle cell migration and apoptosis in spiral artery remodeling. Journal of Cellular Biochemistry. 2019;120:15997-6007.

104. Liu J, Shu B, Zhou ZH, Xu YB, Liu YL, Wang P, et al.: Involvement of miRNA203 in the proliferation of epidermal stem cells during the process of DM chronic wound healing through Wnt signal pathways. Stem Cell Research \& Therapy. 11, (2020).

105. Peng X, Lai KS, She P, Kang J, Wang T, Li G, et al. Induction of Wnt signaling antagonists and p21activated kinase enhances cardiomyocyte proliferation during zebrafish heart regeneration. Journal of molecular cell biology. 2021;13:41-58. 
106. Ehyai S, Miyake T, Williams D, Vinayak J, Bayfield MA, McDermott JC. FMRP recruitment of betacatenin to the translation pre-initiation complex represses translation. Embo Reports. 19, (2018).

107. Yang F, Huang D, Xu L, Xu W, Yi X, Zhou X, et al.: Wnt antagonist secreted frizzled-related protein I (sFRP1) may be involved in the osteogenic differentiation of periodontal ligament cells in chronic apical periodontitis. Int Endod J. (2021).

108. Petersen PH, Zou K, Hwang JK, Jan YN, Zhong W. Progenitor cell maintenance requires numb and numblike during mouse neurogenesis. Nature. 2002;419:929-34.

109. Liang M, Guo QY, Huang FZ, Han GF, Song K, Luo JL, et al. Notch signaling in bone marrow-derived FSP-1 cells initiates neointima formation in arteriovenous fistulas. Kidney Int. 2019;95:1347-58.

110. Jia S, Ma WD, Zhang CY, Zhang Y, Yao ZH, Quan XH, et al. Tanshinone IIA attenuates high glucose induced human VSMC proliferation and migration through miR-21-5p-mediated tropomyosin 1 downregulation. Arch Biochem Biophys. 2019;677:108154.

111. Han H, Zhan Z, Xu J, Song Z. TMEFF2 inhibits pancreatic cancer cells proliferation, migration, and invasion by suppressing phosphorylation of the MAPK signaling pathway. Onco Targets Ther. 2019;12:11371-82.

112. Brunner PM, Heier PC, Mihaly-Bison J, Priglinger U, Binder BR, Prager GW. Density enhanced phosphatase-1 down-regulates urokinase receptor surface expression in confluent endothelial cells. Blood. 2011;117:4154-61.

113. Sun Y, Li S, Yu W, Chen C, Liu T, Li L, et al. CD148 Serves as a Prognostic Marker of Gastric Cancer and Hinders Tumor Progression by Dephosphorylating EGFR. J Cancer. 2020;11:2667-78.

114. Park HS, Quan KT, Han JH, Jung SH, Lee DH, Jo E, et al. Rubiarbonone C inhibits platelet-derived growth factor-induced proliferation and migration of vascular smooth muscle cells through the focal adhesion kinase, MAPK and STAT3 Tyr705 signalling pathways. Brit J Pharmacol. 2017;174:414054.

115. Cattaruzza M, Schafer K, Hecker M. Cytokine-induced down-regulation of zfm1/splicing factor-1 promotes smooth muscle cell proliferation. J Biol Chem. 2002;277:6582-9.

116. Zuo C, Li X, Huang J, Chen D, Ji K, Yang Y, et al. Osteoglycin attenuates cardiac fibrosis by suppressing cardiac myofibroblast proliferation and migration through antagonizing lysophosphatidic acid 3/matrix metalloproteinase 2/epidermal growth factor receptor signalling. Cardiovasc Res. 2018;114:703-12.

117. Guo X, Chen KH, Guo Y, Liao H, Tang J, Xiao RP. Mitofusin 2 triggers vascular smooth muscle cell apoptosis via mitochondrial death pathway. Circ Res. 2007;101:1113-22.

118. Tsaousi A, Williams H, Lyon CA, Taylor V, Swain A, Johnson JL, et al. Wnt4/beta-catenin signaling induces VSMC proliferation and is associated with intimal thickening. Circ Res. 2011;108:427-36.

119. Shitashige M, Satow R, Honda K, Ono M, Hirohashi S, Yamada T. Increased susceptibility of Sf1(+/-) mice to azoxymethane-induced colon tumorigenesis. Cancer Sci. 2007;98:1862-7.

120. Xu T, Zhang R, Dong M, Zhang Z, Li H, Zhan C, et al. Osteoglycin (OGN) Inhibits Cell Proliferation and Invasiveness in Breast Cancer via PI3K/Akt/mTOR Signaling Pathway. Onco Targets Ther. 
2019;12:10639-50.

121. Wu M, Peng S, Yang J, Tu Z, Cai X, Cai CL, et al. Baf250a orchestrates an epigenetic pathway to repress the Nkx2.5-directed contractile cardiomyocyte program in the sinoatrial node. Cell Res. 2014;24:1201-13.

122. Curran J, Makara MA, Little SC, Musa H, Liu B, Wu X, et al. EHD3-dependent endosome pathway regulates cardiac membrane excitability and physiology. Circ Res. 2014;115:68-78.

123. Chambers JC, Zhao J, Terracciano CM, Bezzina CR, Zhang W, Kaba R, et al. Genetic variation in SCN10A influences cardiac conduction. Nat Genet. 2010;42:149-52.

124. Feng $S$, Gao L, Zhang D, Tian X, Kong L, Shi H, et al. MiR-93 regulates vascular smooth muscle cell proliferation, and neointimal formation through targeting Mfn2. Int J Biol Sci. 2019;15:2615-26.

125. Qin L, Yang W, Wang YX, Wang ZJ, Li CC, Li M, et al. MicroRNA-497 promotes proliferation and inhibits apoptosis of cardiomyocytes through the downregulation of Mfn2 in a mouse model of myocardial ischemia-reperfusion injury. Biomed Pharmacother. 2018;105:103-14.

\section{Figures}

a

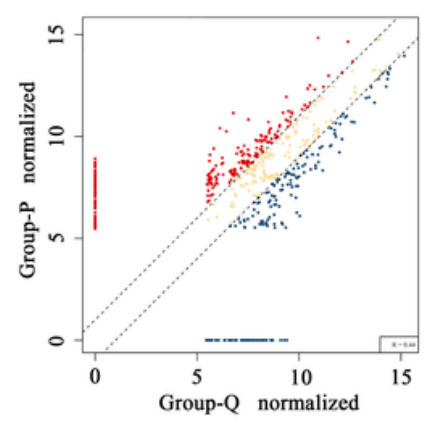

d

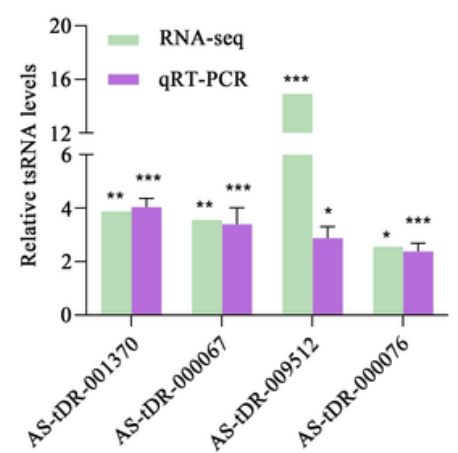

b

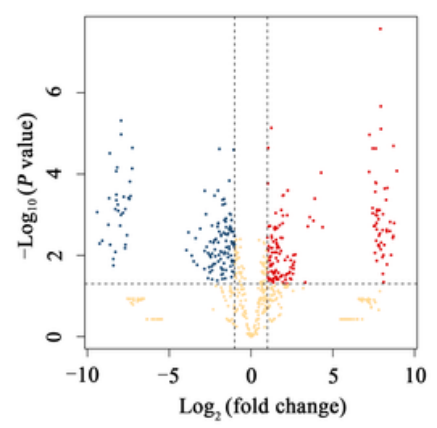

e

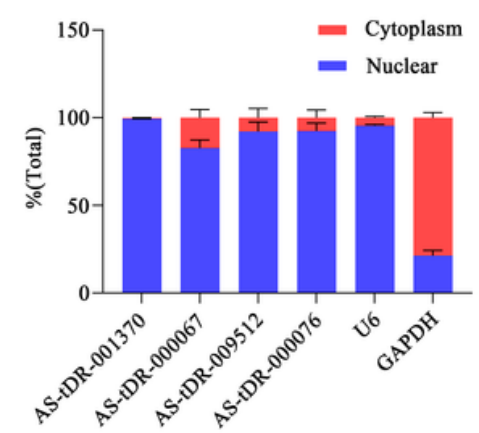

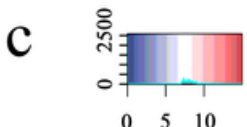

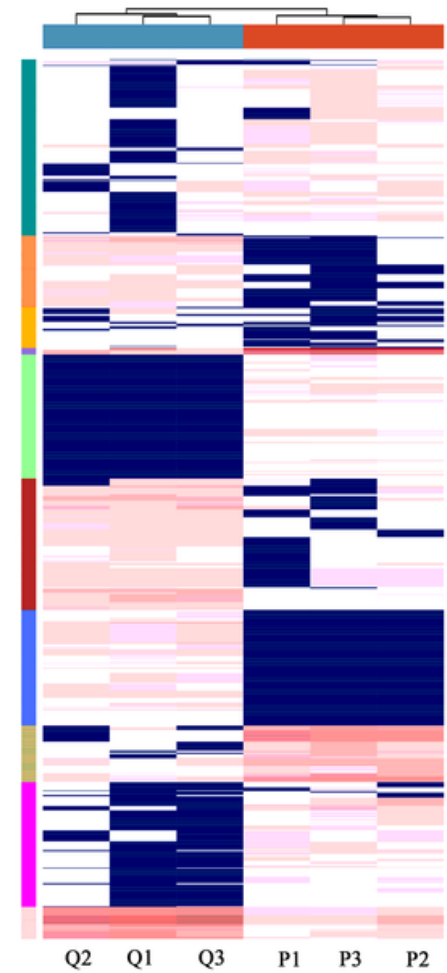

Figure 1 
Expression profile of DEtsRNAs in proliferative and quiescent HASMCs. a Scatter plot shows the DEtsRNAs between proliferative and quiescent groups. X-axis and $Y$-axis values in the scatter plot are the average TPM (Transcripts Per Million) values (log2 scaled) of each group. Genes above the top line (red dots, up-regulation) or below the bottom line (green dots, down-regulation) indicate more than 2.0 fold change (default fold change value is 2.0 ) between two compared groups. Gray dots indicate tsRNAs without differential expression. $b$ Volcano plot describes the DEtsRNAs between proliferative and quiescent groups. Values of the X-axis and Y-axis are fold change (log2-transformed) and p-value (-log10transformed) between two groups. DEtsRNAs with statistical difference and more than 2.0 fold change are indicated by the red dots (up-regulation) or green dots (down-regulation); tsRNAs without statistical difference are indicated by the gray dots. c Hierarchical clustering was performed to analyze the expression of DEtsRNAs between proliferative and quiescent groups. Samples were arranged based on the coefficient of variation (CV) of tsRNAs on the TPM count, which facilitated the assumption of the relationship between them. The color in the panel represents the relative expression level (log2transformed). Color scale is shown above: blue represents an expression level below the mean; red represents an expression level above the mean. P1, P2, and P3 represent the proliferative group; Q4, Q5, and Q6 represent the quiescent group. $d$ Expression levels of DEtsRNAs (AS-tDR-001370, AS-tDR-000067, AS-tDR-009512, and AS-tDR-000076) in proliferative and quiescent HASMCs were validated by qRT-PCR, and results were consistent with sequencing. Control primers: hsa-U6. e DEtsRNAs (AS-tDR-001370, AStDR-000067, AS-tDR-009512, and AS-tDR-000076) are mostly located in the nucleus, which was confirmed by nuclear and cytoplasmic tsRNAs Fractionation experiment. U6 and GAPDH were applied as positive controls for the nucleus and cytoplasm, respectively. The results presented are representative of three independent experiments; bars: $S D .{ }^{*} P<0.05,{ }^{*} \mathrm{P}<0.01,{ }^{\star *} \mathrm{P}<0.001$. 
$\mathrm{a}$

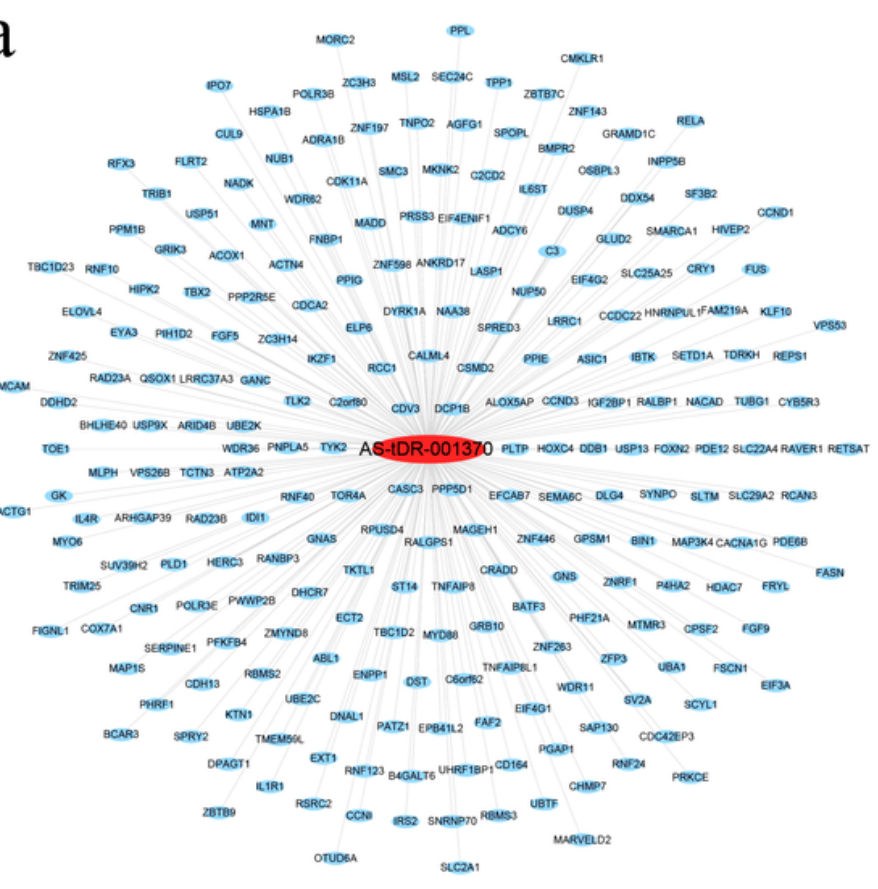

$\mathrm{C}$

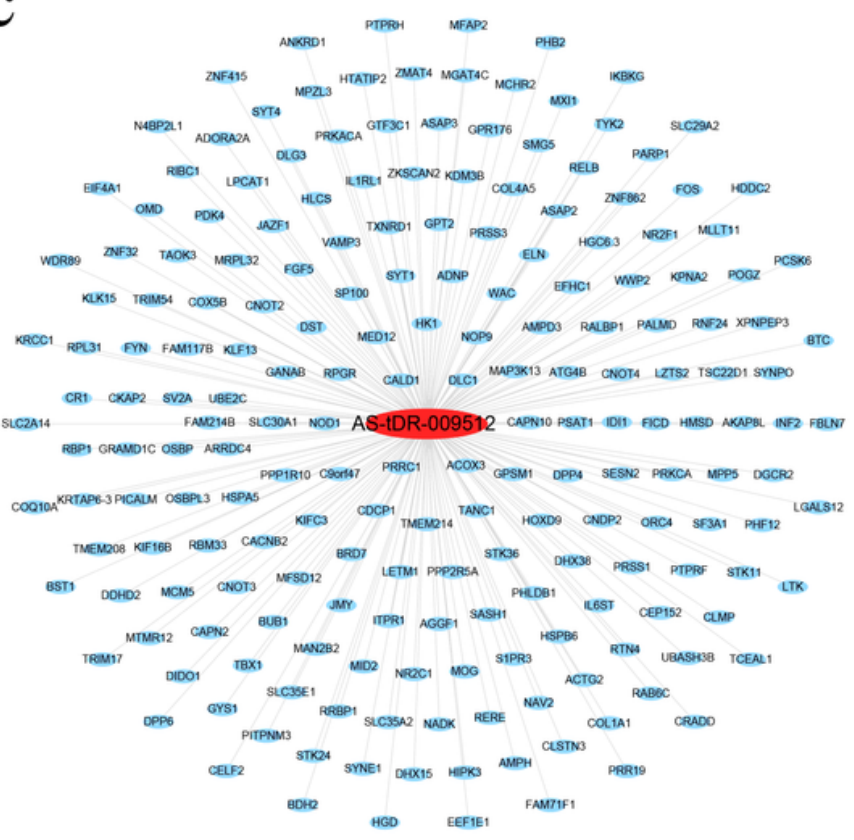

$\mathrm{b}$

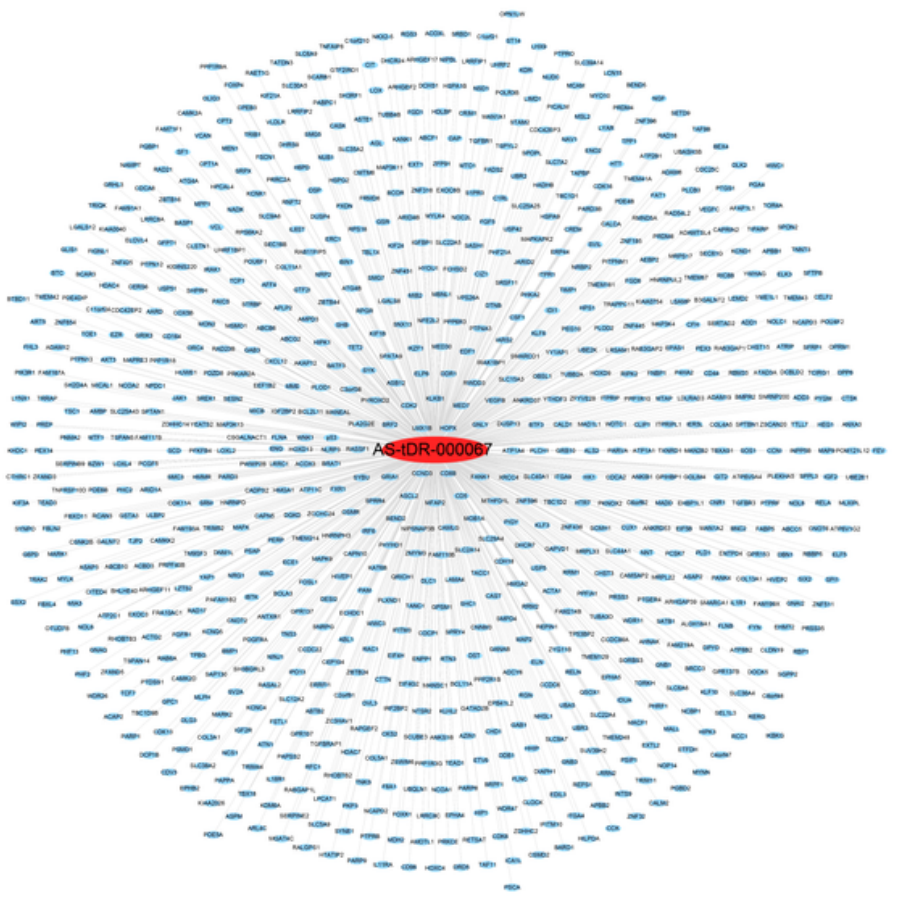

d

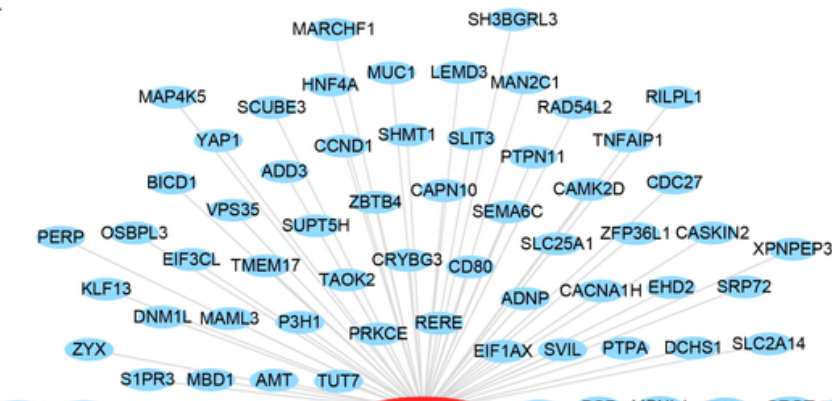

SEC23A TGFB1 AS-tDR-000076 LAMC2 DSP MBNL1 EYA3 CROT RPL28

MANEAL SOS1 SETD1A C6orf15

HERPUD1 WASHC5 PLA2G2E SETMAR KAT6B CCND2
CNOT1 PYROXD2 PRKX BCDIN3D BCR GPC1 CNOT1 PYROXD2 PRKX COPS2 GPATCH8 ZNF596 ST3GAL1

MAPK9 SYNRG PNMA8A GNB1 C17orf8 SCRG1 TIMP2 POFUT1 RBM28 PREX1

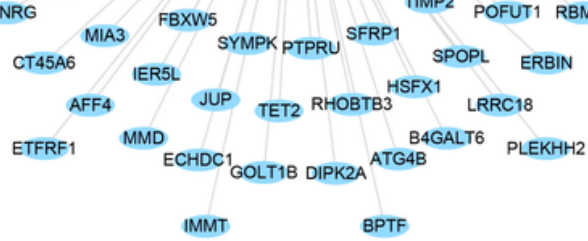

\section{Figure 2}

Interaction networks between DEtsRNAs and related genes containing target promoters in proliferative HASMCs. Subnetworks of (a) AS-tDR-001370, (b) AS-tDR-000067, (c) AS-tDR-009512, and (d) AS-tDR000076. Red nodes: DEtsRNAs; blue nodes: genes containing target promoters. 
a

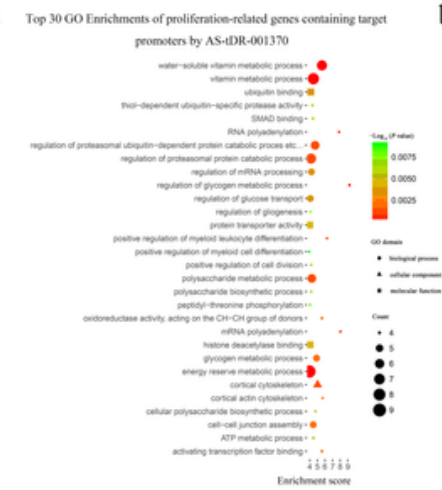

c

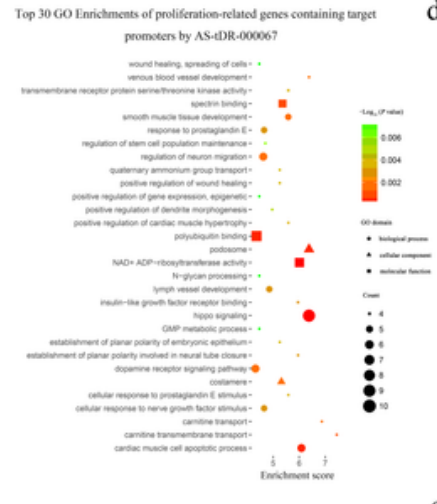

e

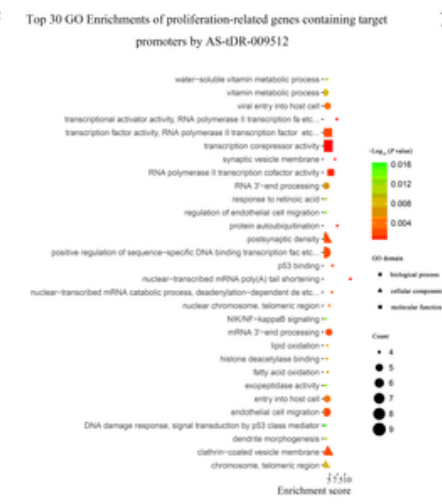

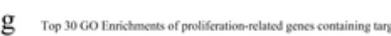
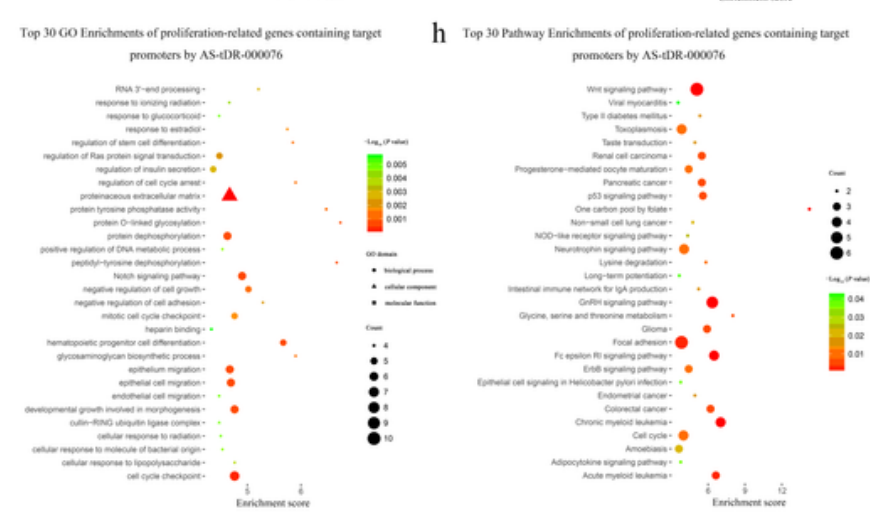

b Top 30 Pathway Enrichments of proliferatio--retated genes containing taryet

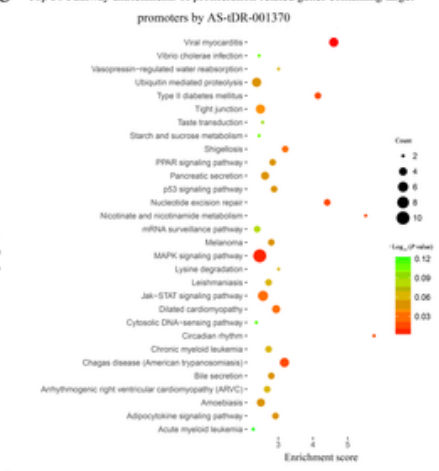

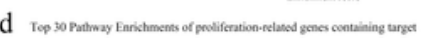

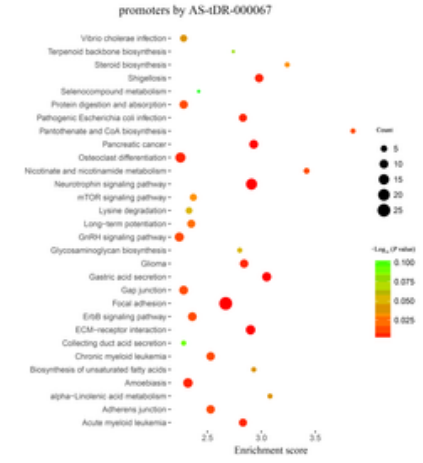

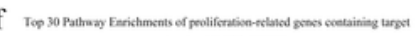

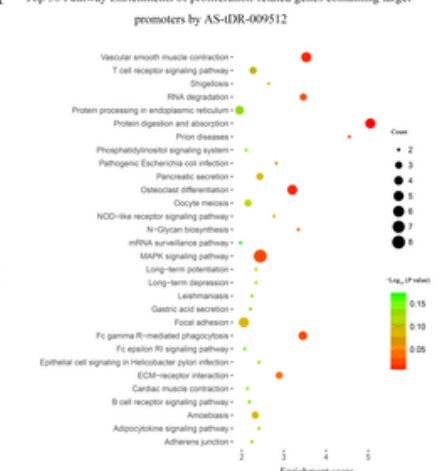

Top 30 Pathway Enrictments of protiferation -related genes containing target

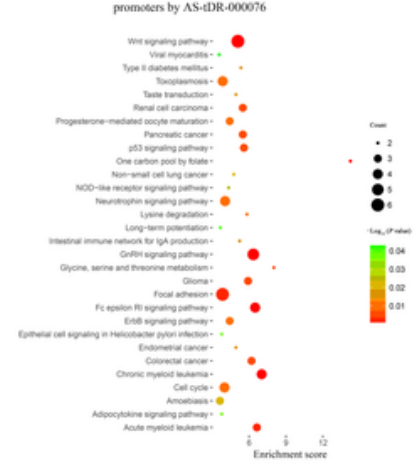

Figure 3

GO and KEGG analyses of genes containing target promoters of DEtsRNAs. Top $30 \mathrm{GO}$ terms of genes containing target promoters of (a) AS-tDR-001370, (c) AS-tDR-000067, (e) AS-tDR-009512, and (g) AStDR-000076. Top 30 pathways of genes containing target promoters of (b) AS-tDR-001370, (d) AS-tDR000067, (f) AS-tDR-009512, and (h) AS-tDR-000076. 
a

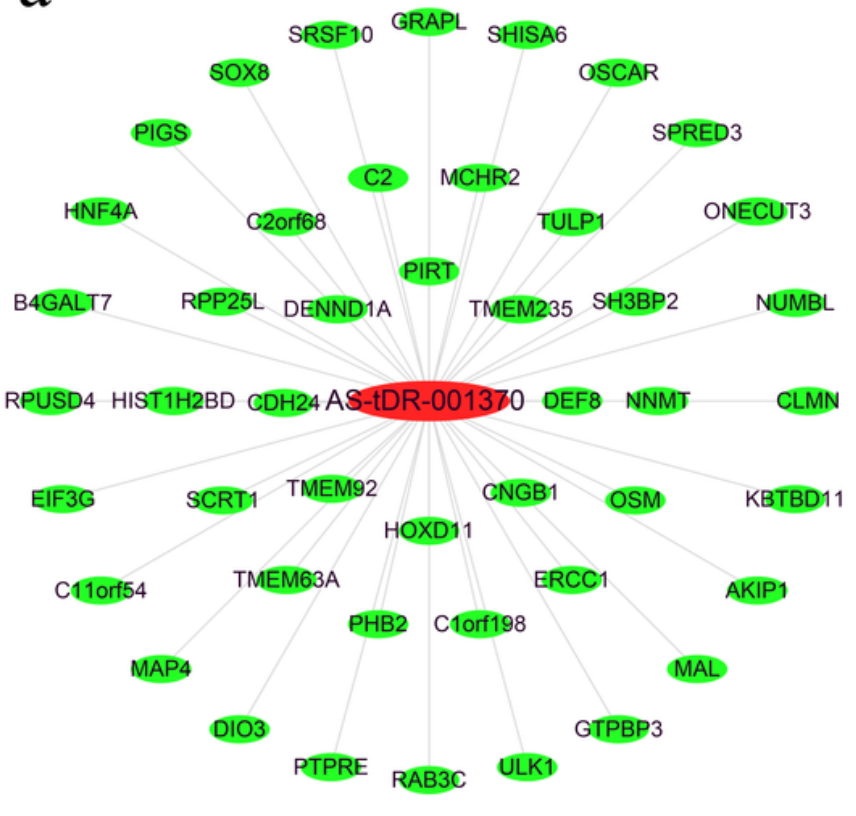

c

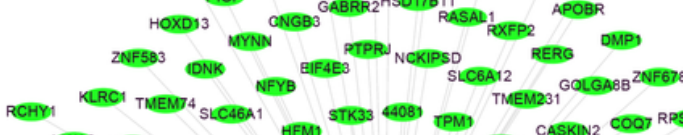
WWA2 KLRC2 RNMT ADSSL1 HFM STK33 4083) TPM) TMEM231 ACER3 CASKIN2 COQ7 RPSERC1 AGA

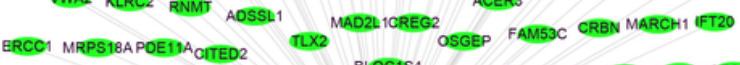
FAM3B PRH2 LMCD1 MX2

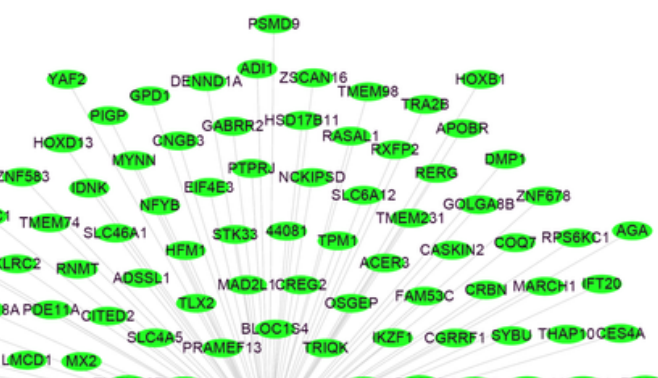
TM

SYPL1 ACSM3 LRRCBBRCSK6 CAMLG SIX4 VSTM2A ZMATA KIRREL2 ASTN2 ARHGEF9 UPRT AS-tDR-009512 PVR SP3 ELF5 KRT34 CTCI TBX5 POLR2C RCSK2 HPGDSCDCA2ATP2B3 RLN2

MTMR6 SYNPO2 ZMAT2 HEPH ZC3H12B MFN2 TBXAS1 ODN GHSR NMRB1 GHMP3 CINP 44082 TECTB

NI15 NATBL NXT2 NG3 GPXY MAPG KNGI ARB CYP2C8 SEC23IP MYCT1 DDIT3 CHX9 WEIKKN2

SH2D7 HCN3 SCN10A NFASC TYRP1 STAP1 NPY GOLGABA ARAIP AMDHD1 C10L LRRN3 TMEFE2

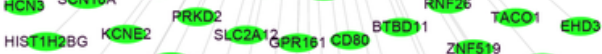

ZMYM3 PTCHD1 ${ }_{\text {ORC6 }}$ TTC33 ABLIM3 TRIM5 BCL113A SNF519 SNTN

SEMABO RCDH1 GPR183 SUMO2 BEND2 HEBP2 HSD1787

LRIGI b

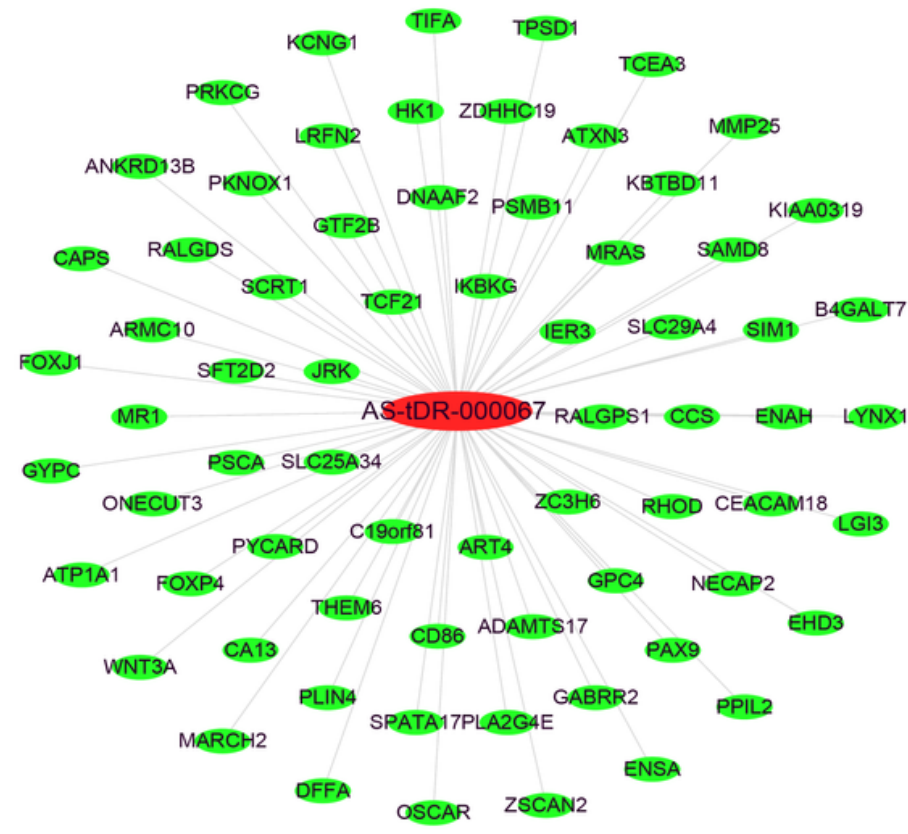

d

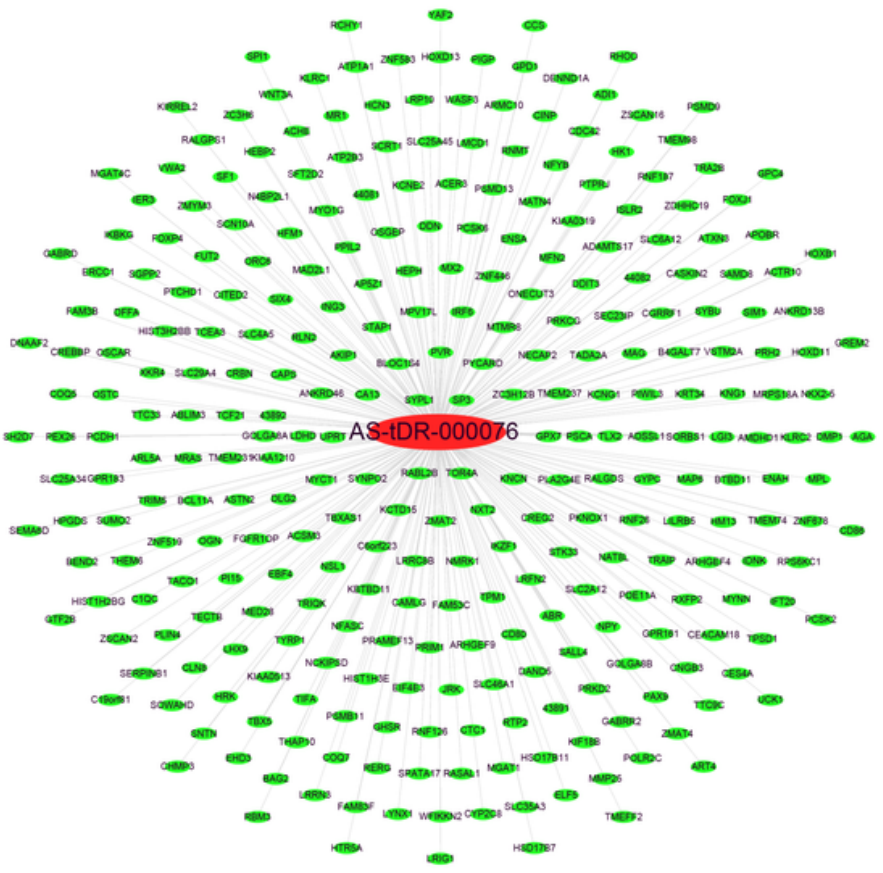

\section{Figure 4}

Interaction networks between DEtsRNAs and related target DEmRNAs in proliferative HASMCs.

Subnetworks of (a) AS-tDR-001370, (b) AS-tDR-000067, (c) AS-tDR-009512, and (d) AS-tDR-000076. Red nodes: up-regulated DEtsRNAs; green nodes: down-regulated target DEmRNAs. 

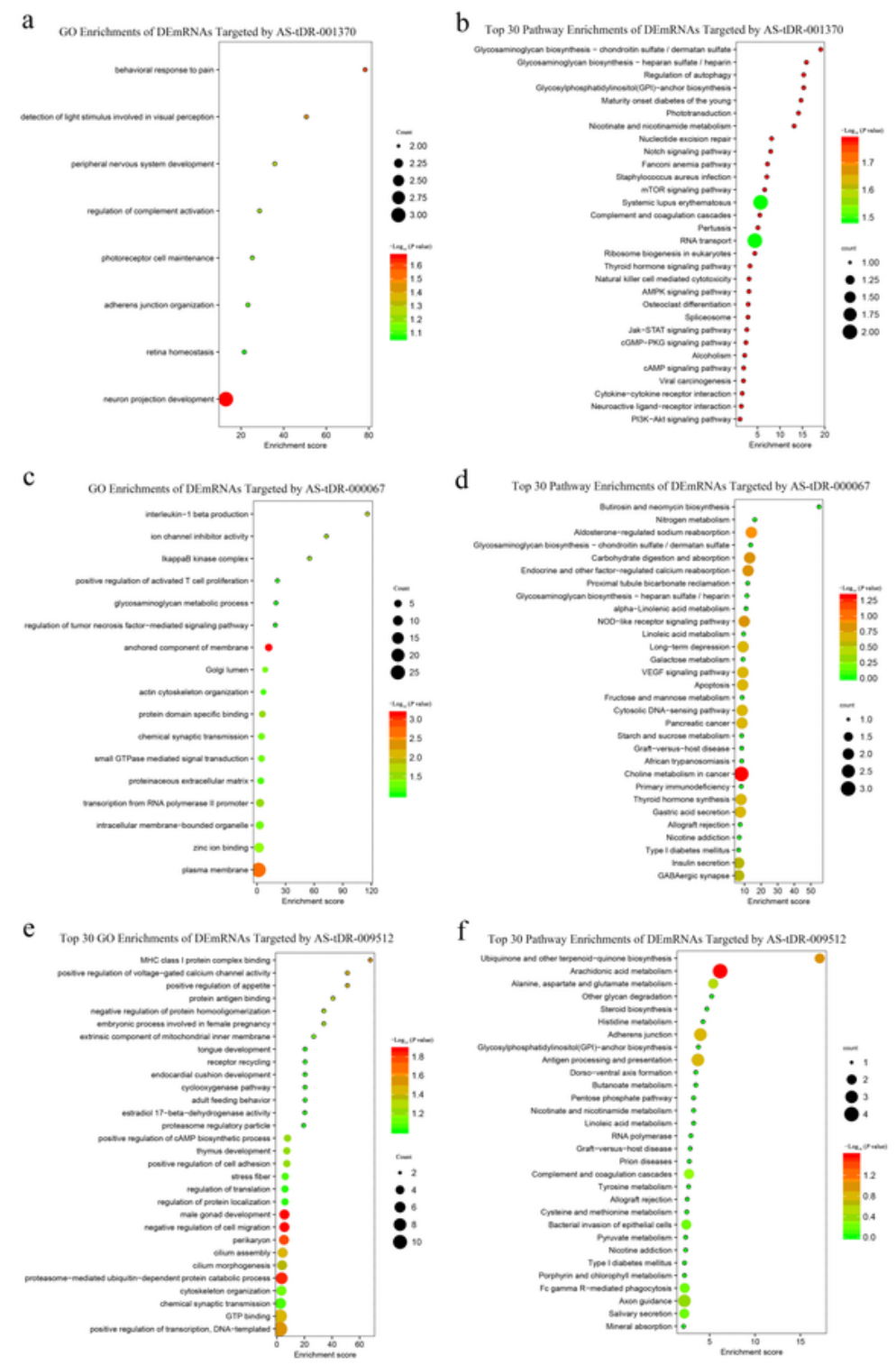

f Top 30 Pathuay Enrichments of DEmRNAs Targeced by AS-1DR-009512
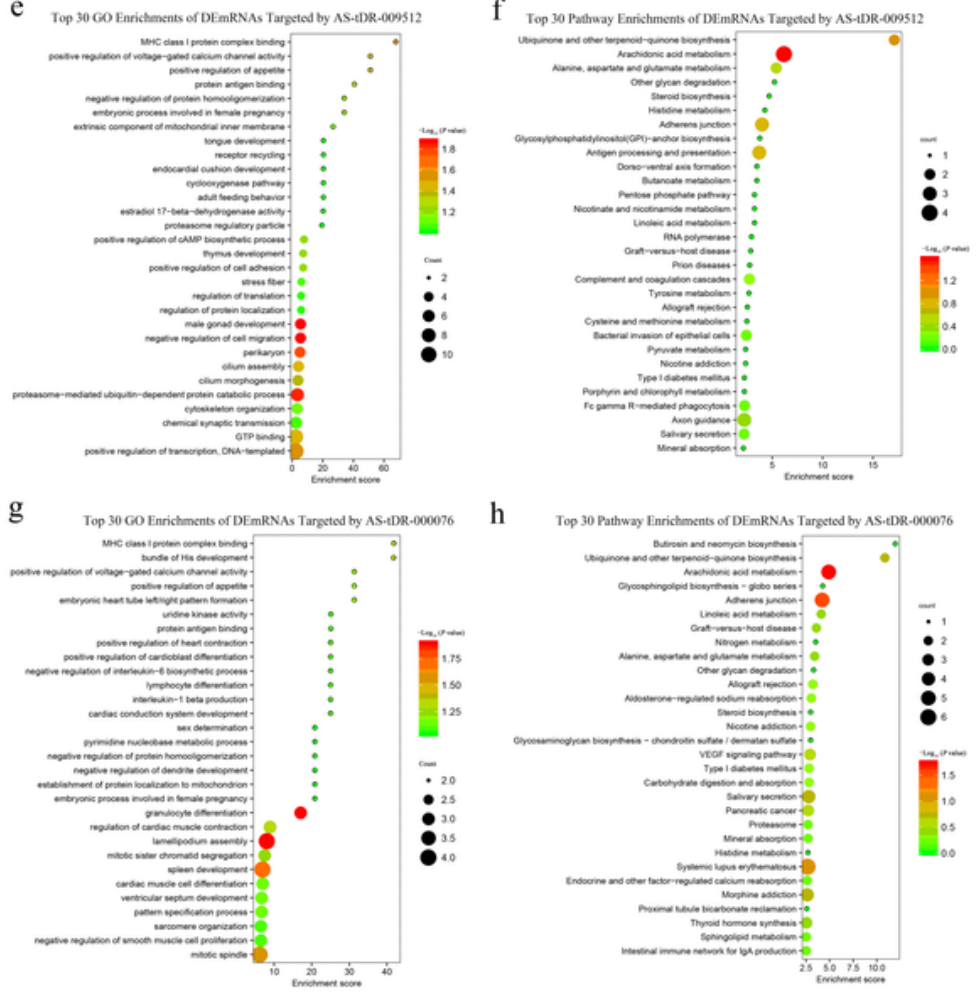

\section{Figure 5}

GO and KEGG analyses of target DEmRNAs of DEtsRNAs. GO terms of target DEmRNAs of (a) AS-tDR001370, (c) AS-tDR-000067, (e) AS-tDR-009512, and (g) AS-tDR-000076. Pathways of target mRNAs of (b) AS-tDR-001370, (d) AS-tDR-000067, (f) AS-tDR-009512, and (h) AS-tDR-000076. 
a

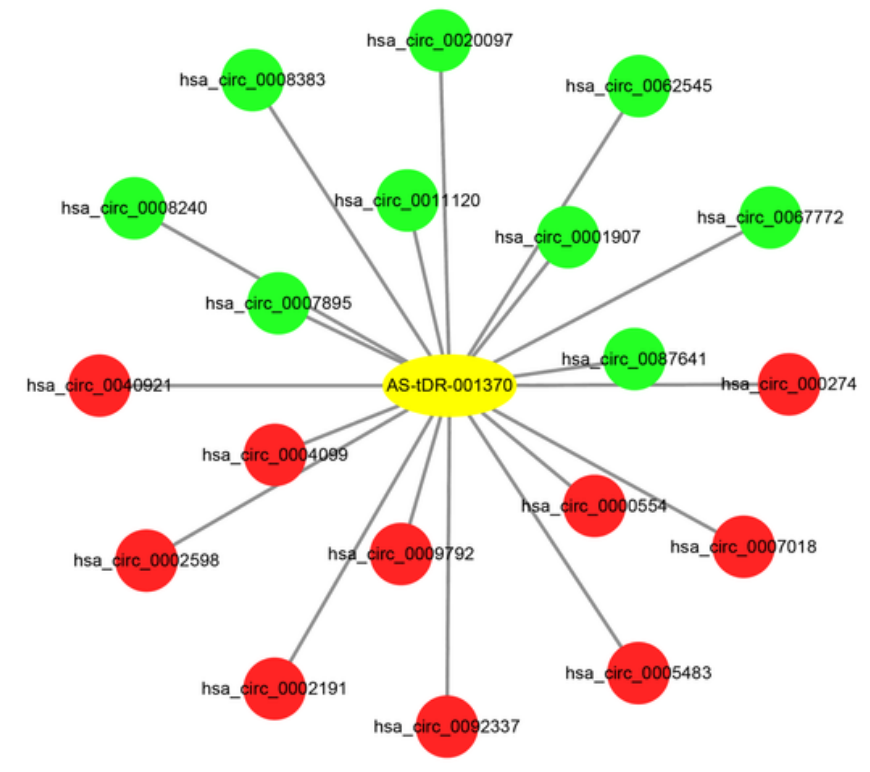

$\mathrm{C}$

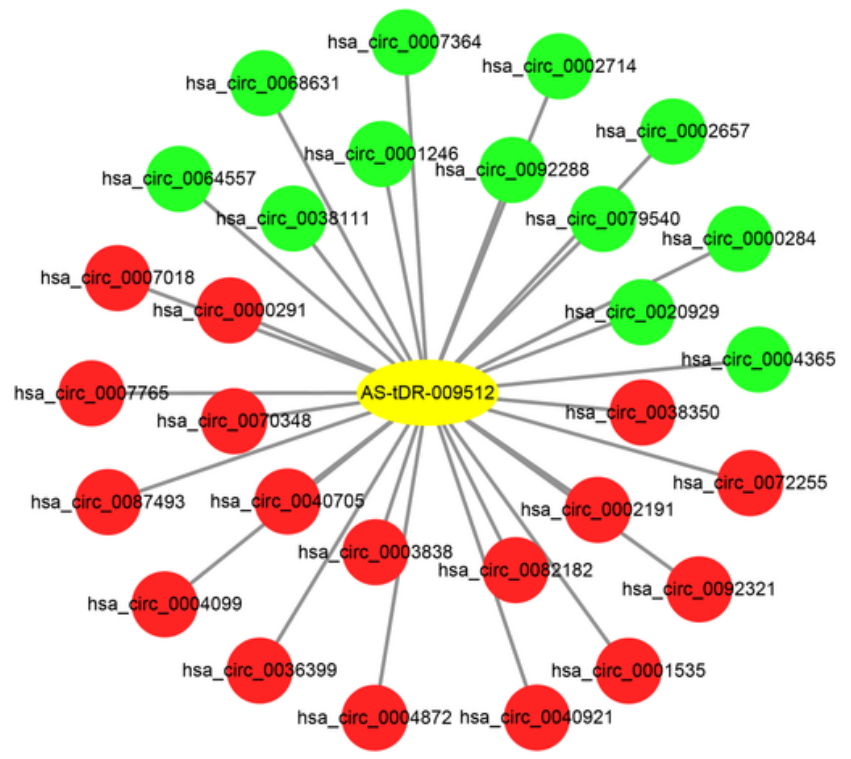

b

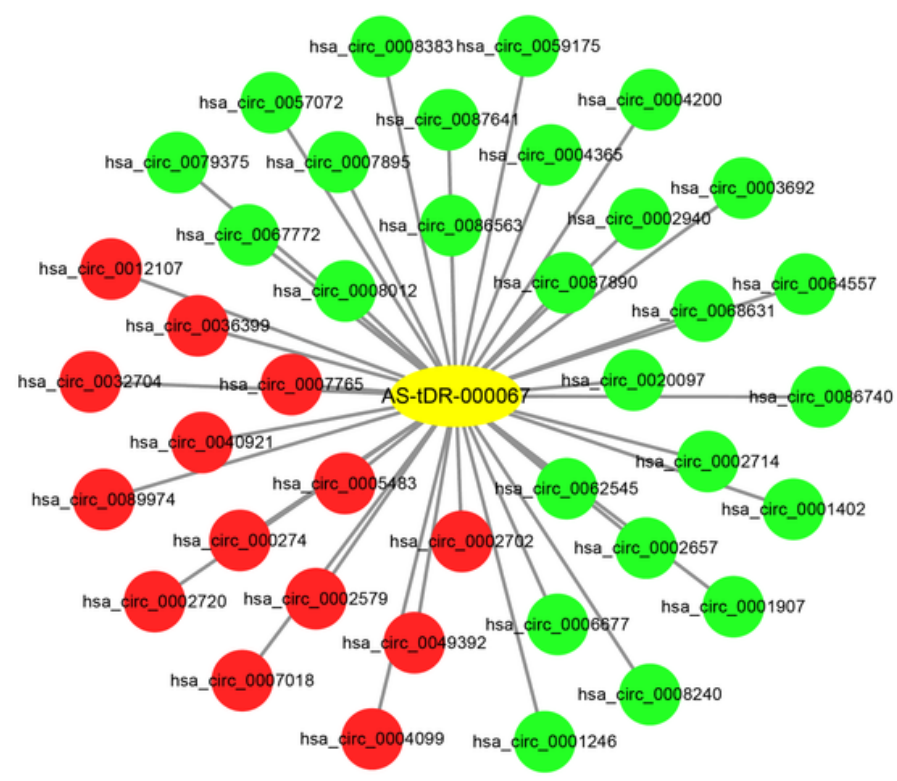

d

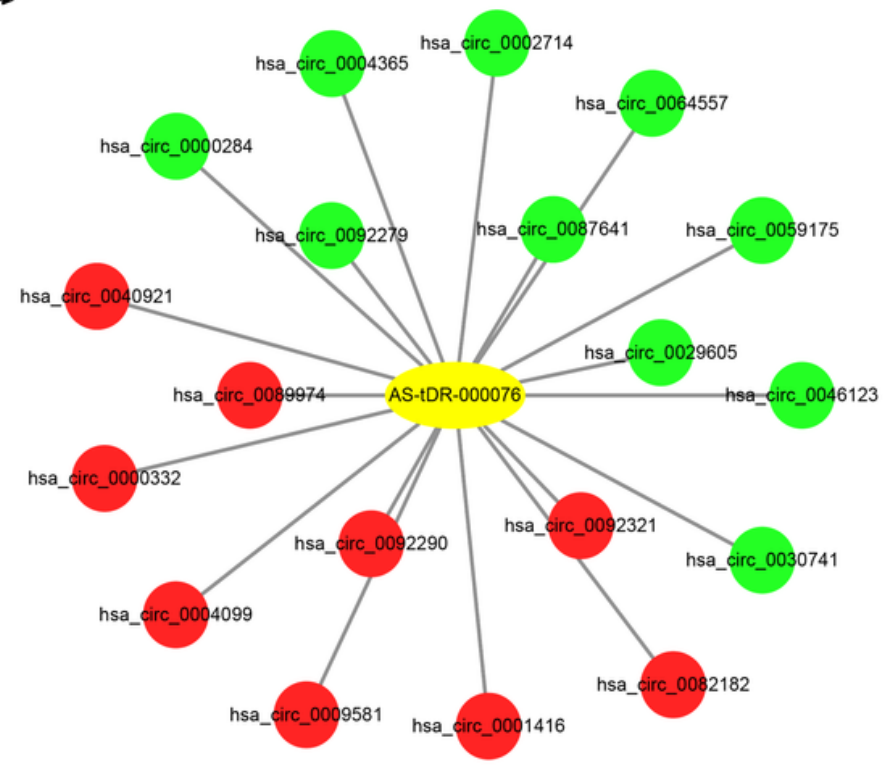

\section{Figure 6}

Interaction networks between DEtsRNAs and related target DEcircRNAs in proliferative HASMCs. Subnetworks of (a) AS-tDR-001370, (b) AS-tDR-000067, (c) AS-tDR-009512, and (d) AS-tDR-000076. Yellow nodes: up-regulated DEtsRNAs; red nodes: up-regulated target DEcircRNAs; green nodes: downregulated target DEcircRNAs. 
a
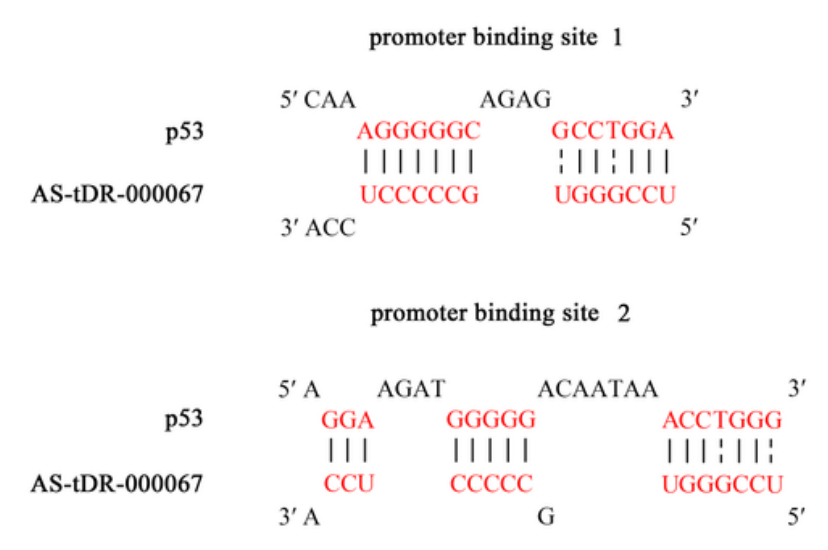

d
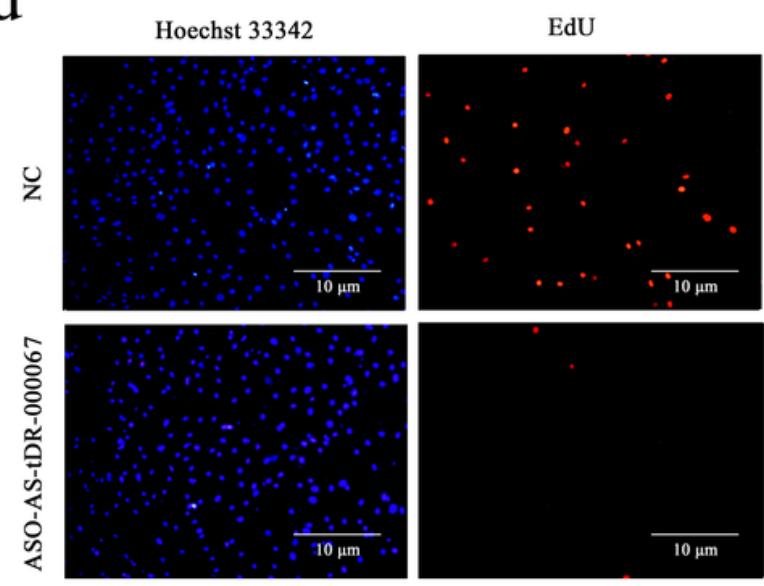

b

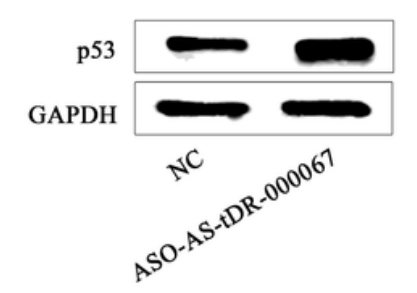

$\mathrm{C}$

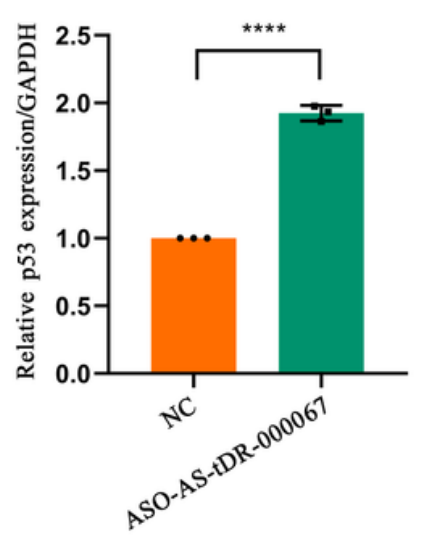

e

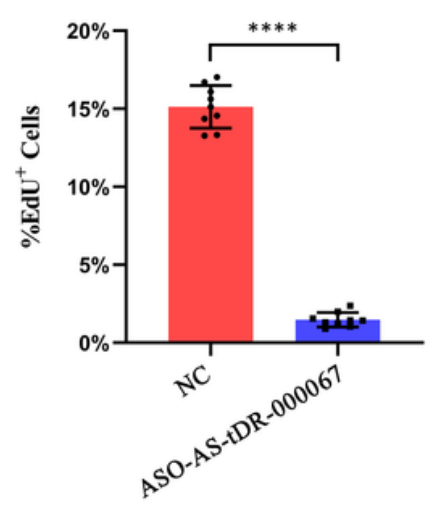

Figure 7

Knockdown of AS-tDR-000067 inhibits the proliferation of HASMCs. a The combination of AS-tDR000067 with the promoter of $p 53$ depends on 14 and/or 15 bp base pairing. $b$ By western blot analysis, the expression of p53 was up-regulated in proliferative HASMCs transfected with ASO-AS-tDR-000067, comparing with NC. c Based on the NC group, the protein expression level of p53 detected by Western blot was analyzed. $d$ EdU was used to detect HASMC proliferation viability. Red dots indicate EdU positive cells, blue dots indicate live cells stained with Hoechst 33342. e The percentage of EdU positive cells is in (d). The results presented are representative of three independent experiments; bars: $S D$. ${ }^{*} P<0.05, \star \star P<$ $0.01, * \star * P<0.001$. 
a

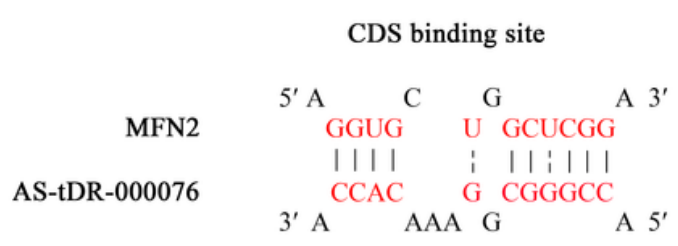

3'-UTR binding site

MFN2

5' CAUG A G C $3^{\prime}$

|। | | | | : | |

CAA GG CGGGCC

$3^{\prime}$ ACCA A

A $5^{\prime}$

d

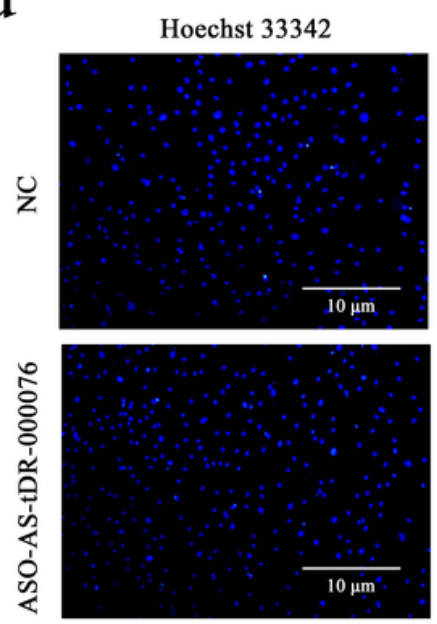

b

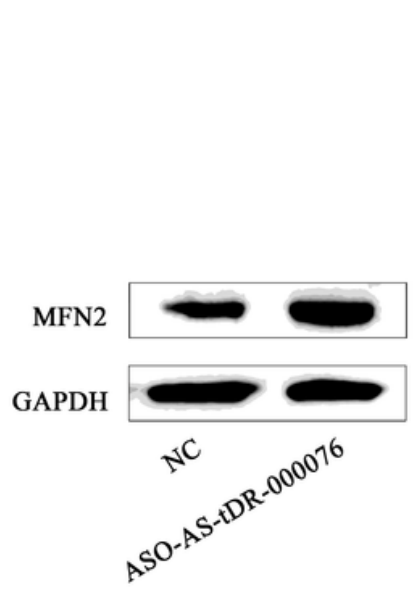

$\mathrm{C}$

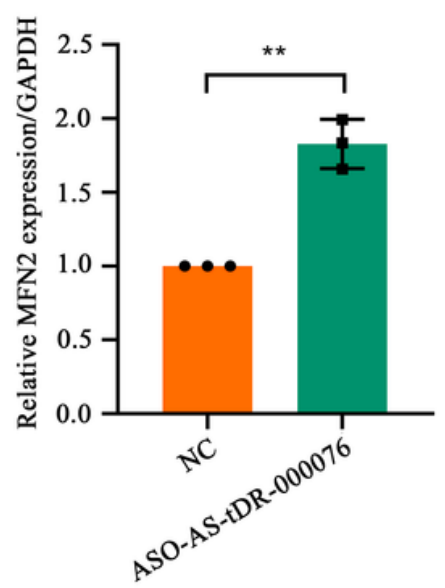

e

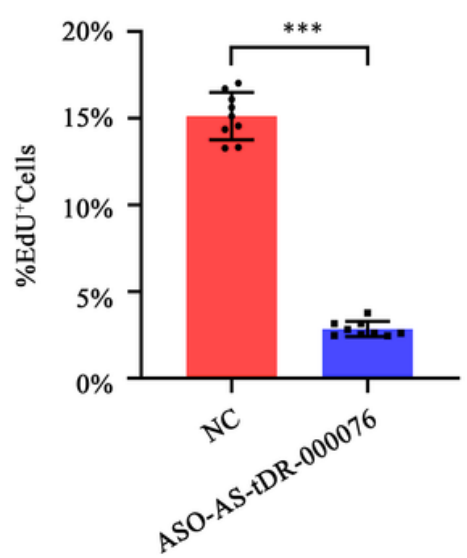

\section{Figure 8}

Knockdown of AS-tDR-000076 inhibits the proliferation of HASMCs. a The combination of AS-tDR000076 with CDS and 3'-UTR of MFN2 depends on 11 bp base pairing. b By western blot analysis, the expression of MFN2 was up-regulated in proliferative HASMCs transfected with ASO-AS-tDR-000076, comparing with NC. c Based on the NC group, the protein expression level of MFN2 detected by Western blot was analyzed. $d$ EdU was used to detect HASMC proliferation viability. Red dots indicate EdU positive cells, blue dots indicate live cells stained with Hoechst 33342. e The percentage of EdU positive cells is in (d). The results presented are representative of three independent experiments; bars: $S D$. ${ }^{*} P<0.05, * * P<$ $0.01, * * * P<0.001$.

\section{Supplementary Files}

This is a list of supplementary files associated with this preprint. Click to download.

- SupplementaryFigure1.tif 
- SupplementaryFigure2.tif

- SupplementaryTable1.docx

- SupplementaryTable2.docx 